\title{
Sprachkonzepte in der Öffentlichkeit
}

\section{Kognitive Repräsentationen der deutschen Sprache}

Zusammenfassung: Der folgende Beitrag behandelt Sprachkonzepte in der Öffentlichkeit. Im Zentrum steht dabei die Frage, wie Sprecherinnen und Sprecher bzw. linguistische Laien ihre eigene Sprache, das Deutsche, konzeptualisieren. Dafür umreißt der Beitrag in einem ersten Schritt grundlegende Konzepte wie Öffentlichkeit, Alltag und Wissen. Anschließend wird die Laienlinguistik als kognitionslinguistische Angelegenheit beschrieben und die Theorie des Epistemikons expliziert. Der empirische Teil stellt dann einen exemplarischen Ausschnitt einer Studie dar, bei der die Gewährspersonen (GP) auf den nominalen Stimulus ,Deutsche Sprache' reagieren. Es stehen dabei vier Konzepte im Zentrum: VARIATION, TRADITION und Norm bzw. STANDARDISIERUNG, die allerdings von den Gewährspersonen jeweils unterschiedlich semantisch gefüllt werden.

Abstract: The following article deals with language concepts in the public sphere. The focus lies on the question how speakers (or linguistic laypersons) conceptualize their own language - the German language. For this purpose, the article initially outlines basic concepts such as public sphere, everyday life and knowledge. This is followed by a description of lay linguistics as a cognitive-linguistic matter and an explanation of the theory of the epistemicon. The empirical part then presents an excerpt from a study in which subjects react to the nominal stimulus 'German language'. Four concepts are central in this matter: VARIATION, TRADITION, and NORM resp. STANDARDIZATION - these concepts, however, are filled differently by the subjects in terms of semantics.

Schlüsselwörter: Alltag, Konzept, Laie, Laienlinguistik, Metasprachwissen, Öffentlichkeit, kognitive Semantik, Spracheinstellungen, Sprachkonzepte, Sprachreflexivität, Sprachwissen

\footnotetext{
Anmerkung: Ich danke Ann-Katrin Nöhren vom Projekt FrameNet und Konstruktikon des Deutschen (Düsseldorf) sowie Verena Sauer (Kiel) für zahlreiche Kommentare zu einer früheren Version des Beitrags.
}

Hoffmeister, Toke: Germanistisches Seminar, Christian-Albrechts-Universität zu Kiel, Leibnizstraße 8, 24118 Kiel, hoffmeister@germsem.uni-kiel.de 


\section{Einleitende Bemerkungen}

Die Sprache stellt semantische Felder oder Sinnzonen her, die wiederum durch Sprache abgegrenzt werden. Vokabular, Grammatik und Syntax sorgen für Gliederung und Ordnung im Zuständigkeitsbereich der Semantik.

(Berger \& Luckmann 2013: 42)

Seit etwa den 1980er Jahren (vgl. für die Germanistik z. B. Kremer 1984; Mattheier 1985; international z. B. Preston 1982) hat sich innerhalb der Linguistik eine Form des Bewusstseinswandels (zunächst in Bezug auf Dialekte) vollzogen. Infolgedessen werden nicht mehr bloß fachlinguistische Herangehensweisen berücksichtigt, sondern auch ein öffentlicher, d. h. laikaler Blick auf Sprache als relevant anerkannt. Dennoch hat die sog. Laienlinguistik in einigen Bereichen noch Akzeptanzprobleme, die schon bei Hoenigswald (1966: 20) thematisiert werden. Im Anschluss daran hat sich in einschlägigen Forschungsarbeiten die Ansicht etabliert, dass es bei laikalen metasprachlichen (bzw. metapragmatischen, vgl. Spitzmüller 2019) Äußerungen nicht um fehlerhafte Äußerungen geht, sondern ein eigener (konstruktivistisch-konsensueller) Wahrheitsbegriff formuliert werden muss (vgl. Hoffmeister 2020a; Lehr 2002: 22-41). Derartige Herangehensweisen werden bspw. im Rahmen von subjektiven Theorien ${ }^{1}$ beschrieben (vgl. Hoffmeister 2020b; Paul 2003, zur Kritik des Terminus Theorie z. B. Wilton \& Stegu 2011: 3; zu konstruktivistischen Grundlagen Felder \& Gardt 2018).

Zur Laiensicht auf deutsche Dialekte sind mittlerweile eine ganze Reihe von Forschungsergebnissen vorgelegt worden (vgl. z. B.: Anders 2010; Hundt, Palliwoda \& Schröder 2017; Purschke 2011; Sauer 2018; einen Überblick geben Hundt 2018 sowie Purschke \& Stoeckle 2019). Auch Studien, die sich nicht mit der Wahrnehmung regionaler Varietäten beschäftigen, sondern andere Aspekte von Sprache im Rahmen einer sog. Laienlinguistik (explizit oder implizit) in den Blick nehmen, liegen vor (vgl. u. a. Ankenbrand 2013; Antos 1996; Cuonz 2014; König 2014; Lehr 2002; Paul 1999; Spitzmüller 2005; Strauss 2018; Williams

1 ,Subjektive Theorien' wurden zuerst vom Forschungsprogramm Subjektive Theorien (vgl. Groeben et al. 1988; Groeben \& Scheele 1982) beschrieben. Dabei geht es um die Beschreibung von Gegensätzen zu wissenschaftlichen Theorien, d. h. Vorstellungen von Laien, die sich durch zwar inhaltlich von wissenschaftlichen Erkenntnissen unterscheiden können, aber strukturelle Ähnlichkeiten zu den wissenschaftlichen Theorien aufweisen (Kohärenz, Abgeschlossenheit, Verfestigung etc.). Allerdings geht es nicht darum, subjektive Theorien empirisch zu überprüfen, sie erhalten ihre Legitimation vielfach durch Rechtfertigungsstrategien, Allgemeingültigkeitsansprüche oder auch alltagslogische Schlüsse (vgl. Kienpointner 1992). 
2020; einen Überblick bieten zudem Antos, Niehr \& Spitzmüller 2019 sowie Becker, Herling \& Wochele i. E.). Allerdings gibt es bisher keine Darstellung des Konzeptes DEUTSCHE SPRACHE, das in der Öffentlichkeit vorherrschend ist. Und das, obwohl Berger \& Luckmann (2013: 39) festhalten, dass „[d]as Verständnis des Phänomens Sprache [...] entscheidend für das Verständnis der Wirklichkeit der Alltagswelt“ ist. Mit Faulstich (2008) liegt ein erster Ansatz vor, der aus einer diskursfokussierenden Perspektive mit korpuslinguistischen Methoden ein Konzept HochDEUTSCH im 18. Jahrhundert expliziert. Über rezente Sprachkonzepte in der Öffentlichkeit, die eigentlich die Grundlage für die oben erwähnten Studien zu regionalen Varietäten sein müssten, existiert bisher noch keine Forschung. Allerdings kann Berthele (2002) einen ersten, für den vorliegenden Beitrag fruchtbaren Zugang zu den ,mental models of language، aus rezenter (und kognitionslinguistischer!) Perspektive liefern. Der Ansatz ist jedoch eher summarisch und lässt zentrale theoretische wie empirische Fragestellungen für eine systematische germanistische Laienlinguistik außen vor. ${ }^{2}$ Der vorliegende Beitrag versteht sich also als eine Ergänzung bzw. Fortführung von Bertheles Ansatz und versucht einen Beitrag zur Schließung theoretischer und empirischer Lücken zu leisten. Kognitionssemantische Ansätze, wie sie diesem Beitrag zugrunde liegen, sind von der Laienlinguistik bisher nicht grundlegend berücksichtigt worden. Allerdings gibt es in der Forschung weitere Bestrebungen, dieses Desiderat zu beseitigen (vgl. exemplarisch den Sammelband von Gautier \& Varga [i. Vorb.] mit einem Abschnitt $\mathrm{zu}$,Frames in der ExpertenLaien-Kommunikation').

Der Beitrag geht wie folgt vor: Zunächst werden in Abschnitt 2 die theoretischen Grundlagen des Forschungsparadigmas erläutert. Dort wird insbesondere auf die drei grundlegenden Begriffe Öffentlichkeit, Alltag und Wissen eingegangen. Daran anschließend (Abschnitt 3) werden die Umrisse einer kognitiven Laienlinguistik skizziert. ${ }^{3}$ In Abschnitt 4 wird das Setting einer empirischen

2 So behandelt Berthele zwar zentrale Konzepte wie konzeptuelle Metaphern (Berthele 2002: 29-43), Granularität (Berthele 2002: 44-59) und Einstellungen (Berthele 2002: 27-29), allerdings fehlt die dort angestrebte kognitionslinguistische Fundierung, die den wirkenden Prozessen Rechnung trägt. Auch die Darstellung der vorhandenen metaphorischen Umschreibungen bei der Konzeptualisierung von Sprache fasst einzelne Aspekte zusammen, ohne die Ergebnisse auf einen operationalisierten Ansatz zur kognitionslinguistischen Beschreibung mentaler Sprachmodelle zu heben.

3 Die Laienlinguistik ist in zwei grundlegende methodologische Ansätze unterteilbar, deren Trennung eher analytischer Natur ist und die deshalb in der Forschungspraxis nicht immer klar vorhanden sein muss: (1) kognitionslinguistische Ansätze und (2) diskurslinguistische Ansätze. Die diskurslinguistischen Ansätze (vgl. bspw. Spitzmüller 2005) spielen in dieser 
Studie vorgestellt, das zum Ziel hat, Sprachkonzepte der Öffentlichkeit, d. h. in diesem Fall von ,Laien`4, zu explizieren. Im Rahmen des vorliegenden Beitrages verstehe ich Laien mit Spitzmüller (in diesem Band) als in gleicher Weise wie Experten an Sprache interessiert, aber aus einer anderen sozialen Position heraus agierend. Es geht also um eine Form des ,laikalen Empowerment‘. Dass Öffentlichkeit und Laien hier in einer derart engen Verbindung stehen, heißt natürlich nicht, dass nicht auch die Bildung von anderen Theorien, z. B. durch Experten, in der Öffentlichkeit stattfindet, allerdings findet eine Fokussierung auch solcher Personen statt, die sich nicht professionell im Rahmen ihres Berufes mit Sprache beschäftigen. Schließlich werden erste Ergebnisse der Untersuchung dargestellt. Es folgen in Abschnitt 5 abschließende Bemerkungen sowie eine Diskussion über verbleibende Forschungsdesiderata.

\section{2 Öffentlichkeit, Alltag, Wissen und die Rolle der Sprache}

Neben empirischer Grundlagenforschung stellt die theoretische Fundierung des Forschungsparadigmas das zweite zentrale Anliegen dar. Die Laienlinguistik hat es bisher zumeist versäumt, sich einer umfassenden theoretischen Diskussion zu stellen, sodass es in der Verantwortung aller an den Forschungsdiskursen Beteiligten liegt, dieses Desiderat aufzuarbeiten. Im Folgenden sollen deshalb zunächst die (für diesen Beitrag und die Laienlinguistik insgesamt) grundlegenden interdisziplinären Konzepte umrissen werden. Hierfür kann sich die Laienlinguistik insbesondere (wissens-)soziologischer Arbeiten bedienen. Das gemeinhin vorherrschende sozial-konstruktivistische Verständnis (vgl. z. B. Anders 2010; Beuge 2019; Hundt 2017; Schröder 2019; Strauss 2018) soll danach

\footnotetext{
Arbeit nur am Rande eine Rolle. Insofern wird durch die Verwendung des Terminus Laienlinguistik auch keine Unterscheidung a) in eine metasprachliche Diskurspraxis und b) die wissenschaftliche Untersuchung dieser Diskurse impliziert (vgl. dazu Fingerhuth \& Boas 2018: 23). Wichtig für den vorliegenden Beitrag ist vielmehr, dass Laienlinguistik als eine Linguistik von Laien verstanden wird (vgl. Antos 1996: 3).

4 Mit dem Terminus Laie ist eine gewisse Problematik verbunden (vgl. u. a. Bock \& Antos 2019; Hoffmeister 2019; Kalverkämper 1990, Antos in diesem Band; Spitzmüller in diesem Band). Eine wissenschaftliche Disziplin, deren Hauptuntersuchungsgegenstand (vgl. Titel dieses Bandes) ein derart problematisches Konzept zugrunde liegt, hat die Verpflichtung, sich ausführlich aus theoretischer und empirischer Perspektive damit auseinanderzusetzen.
} 
um eine kognitive Komponente ergänzt werden, um die Laienlinguistik als sozio-kognitive Angelegenheit (vgl. Cuonz 2014) zu konturieren.

\subsection{Die Struktur der Öffentlichkeit aus laienlinguistischer Sicht}

,Die Öffentlichkeit' ist eine der zentralen Kategorien laienlinguistischer Forschung (vgl. Antos, Niehr \& Spitzmüller 2019), wenngleich der Eindruck entstehen könnte, es handele sich bei fachwissenschaftlichem Handeln nicht zugleich auch um öffentliches Handeln. Natürlich handelt auch die Fachwissenschaft öffentlich, dieser Anspruch wird an sie auch durch die Gesellschaft und die Politik herangetragen. ${ }^{5}$ Öffentlichkeit ist vielmehr im Gegensatz zu "Privatheit" „als allgemein zugänglich[]“ (Bock \& Antos 2019: 54) zu verstehen, weshalb nicht zwingend alle Aspekte wissenschaftlichen Handelns in den Bereich der Öffentlichkeit fallen, potentiell aber ,veröffentlichbar' sind. Die Öffentlichkeit kann mit Peters (2007: 56) allgemein als „eine Art Kollektiv mit einer bestimmten Kommunikationsstruktur oder eine Sphäre kommunikativen Handelns mit bestimmten anspruchsvollen Merkmalen und Funktionen" definiert werden. Drei Aspekte sind dabei besonders hervorhebenswürdig: (1) Es handelt sich um „eine Art Kollektiv“, (2) es existiert eine „bestimmte[] Kommunikationsstruktur“ und (3) den jeweiligen Handlungsweisen sind gewisse Merkmale und Funktionen inhärent. Es gibt innerhalb der Öffentlichkeit also gewisse Merkmale, die einend wirken (z. B. die soziale Position oder der Grad der Institutionalisierung). Darüber hinaus läuft die Kommunikation innerhalb des Kollektivs intentional und teleologisch ab, es werden also gewisse Aushandlungsprozesse diskursiviert (vgl. Felder 2013, 2018). Schließlich liegt jeder einzelnen Handlung mindestens ein Merkmal zugrunde; darüber hinaus sind die Handlungen in Bezug auf ihre Funktionen näher bestimmbar. Konkret bedeutet dies: Handlungen können im Hinblick auf Ausführungszeitpunkt, Zielgruppe, Absicht, Konsequenz etc. näher bestimmt werden. Dies gilt in besonderem Maße auch für sprachliche Handlungen ${ }^{6}$, die einen zweifachen Blick ermöglichen. Einerseits

5 Vgl. die Pressemitteilung (122/2019) von Anja Karliczek, Bundesministerin für Bildung und Forschung, vom 18.10.2019: https://www.bmbf.de/de/karliczek-wir-wollen-buergerwissenschaften-als-selbstverstaendliche-forschungsmethode-9930.html (letzter Zugriff: 10.02.2020). 6 Unter sprachlichen Handlungen werden hier keine performativen Akte der Pragmatik verstanden, sondern sprachliche Äußerungen, die durch ihre kontextgebundene Äußerung eine Wirkung in der jeweiligen Lebenswelt haben (vgl. Habermas 1995), wenngleich sie nicht zwin- 
kann eine (linguistische) Fachwissenschaft die (öffentlichen) Sprachhandlungen analysieren, andererseits werden im Alltag (vgl. Abschnitt 2.2) ständig Reflexionen bspw. über Angemessenheit bzw. Akzeptabilität, Grammatikalität (vgl. Hoffmeister 2020b) oder Semantik angestellt (vgl. Paul 2003: 650). Daraus resultiert die Möglichkeit, dass Laien als Teil der Öffentlichkeit ${ }^{7}$ selbst Analysen in Bezug auf die Merkmale und Funktionen von Sprache anstellen. Aus den Überlegungen wird deutlich, dass die Grundvoraussetzung für die Konstitution von Öffentlichkeit eine gemeinsame Sprache darstellt (vgl. Schiewe 2004: 99). Diese Sprache schlägt sich nieder in einer Varietät, die man als Alltagssprache ${ }^{8}$ bezeichnen könnte. Der Terminus produziert gewisse Fragen, nach der Struktur und der Bedeutung der Lebenswelt des Alltags (bzw. der alltäglichen Lebenswelten) für die Öffentlichkeit.

\subsection{Alltägliche Lebenswelten und ihre Sprache(n)}

Die alltäglichen Lebenswelten sind durch Sprache geprägt; Sprache ist überall, sie ist apriorisch (vgl. Gipper 1987). ${ }^{9}$ Insofern geht es um sprachlich formierte

gend z. B. eine rechtliche (z. B. Eheschließung) oder moralische („Ich warne dich!“) Konsequenz besitzen müssen.

7 Natürlich besteht die Öffentlichkeit nicht nur aus Laien. Auch Experten (und andere Personen mit anderen Rollenzuschreibungen) sind Teil der Öffentlichkeit. Das Verhältnis von Laien und Experten innerhalb öffentlicher Räume ist ein komplexes, das hier nicht näher erläutert werden soll (vgl. dazu Bock \& Antos 2019; Spitzmüller in diesem Band; Wichter \& Antos 2001).

8 Etwaige varietätenlinguistische Bedenken (vgl. Felder 2016) an diesem Konzept seien an dieser Stelle hingenommen. Eine Diskussion über Vor- und Nachteile, Angemessenheit oder Zielführung der einzelnen Konzepte wie Alltagssprache, Umgangssprache, Gebrauchssprache, regionaler Standard oder gar Regiolekt etc. soll an dieser Stelle nicht geführt werden. Mit Alltagssprache ist im vorliegenden Kontext also eine Varietät gemeint, die in der Öffentlichkeit in alltäglichen Lebenswelten (vgl. Lehr 2002) gesprochen wird (vgl. Möller \& Elspaß 2015).

9 Dass Sprache apriorisch ist, bezieht sich auf ihre Rolle in Bezug auf das menschliche Denken, Erkennen (vgl. Gipper 1987) und Handeln; es beschreibt die „Bedingung der Möglichkeit aller Erfahrung“ (Gipper 1987: 14) sowie die „unverzichtbare Bedingung der Möglichkeit der [...] erforderlichen gedanklichen Reflexionsprozesse“ (Gipper 1987: 18). Implikationen, die auf eine angeborene Sprachkompetenz (sog. ,Language Acquisition Device‘, vgl. Chomsky 1957, 1959) verweisen, werden an dieser Stelle zurückgewiesen, da hier wie in der rezenten Spracherwerbsforschung die Ansicht vertreten wird, dass das der Spracherwerb eben nicht nach angeborenen Prinzipien und Parametern erfolgt, sondern aus der Entwicklung allgemeiner kognitiver Fähigkeiten resultiert (vgl. Klann-Delius 2016: 83-91). Das Postulat einer Universalgrammatik ist aufgrund der Divergenz der Sprachen ebenso wenig überzeugend (vgl. Evans 
Lebenswelten des Alltags (vgl. Gardt 2001: 34-36; Lehr 2002: 22-41; Schütz \& Luckmann 2003: 659-672). Die einzelnen alltäglichen Lebenswelten unterscheiden sich dabei in ihrem Grad an Öffentlichkeit. Einzelne Ausprägungen können einen hohen Grad an Öffentlichkeit erreichen (z. B. gewisse berufliche Lebenswelten), andere verbleiben tendenziell im Privaten. Alltägliche Lebenswelten bewegen sich in ihrem Öffentlichkeitsgrad also auf einem Kontinuum: Etwas ist mehr oder weniger öffentlich. Das gilt auch für die Prototypizitätsgrade der Öffentlichkeit bestimmter Situationen. Die Individuen entscheiden dabei i. d. R. selbst, welche Elemente sie welchen Öffentlichkeiten (vgl. Bock \& Antos 2019: 55) zugänglich machen wollen. Dafür stehen zwei Methoden zur Verfügung. Möglich ist die Veröffentlichung des Privaten einerseits durch visuelle Methoden (z. B. ein Fotoalbum des letzten Sommerurlaubes oder ein Video der ersten Schritte des Kindes bspw. auf Instagram) und andererseits durch sprachliche Methoden (z. B. Bericht einer Krisensitzung mit der Familie). Beide Methoden können praktisch miteinander verknüpft werden.

Zentral für die Kategorie des Alltags ist die Erfahrung, die auf zwei unterschiedliche Arten möglich ist. Bei der Veröffentlichung des Privaten handelt es sich um die erste Art der Erfahrung, die vermittelte Erfahrung (taught experience). Dabei werden Dritte über eine die Erfahrung vermittelnde Instanz (entweder die erlebende Person selbst oder wiederum eine Person, die die Erfahrung vermittelt erfahren hat) in Kenntnis gesetzt. Der vermittelten Erfahrung ist ein Biasfaktor der verzerrenden Wiedergabe inhärent, da Informationen bei der (mehrfachen) Weitergabe zur Veränderung und Verfälschung neigen. Demgegenüber steht die unvermittelte Erfahrung (direct experience), die an den jeweiligen Körper gebunden ist. Es handelt sich somit um eine Form der embodied experience (vgl. Bengt- sson 2013; Bucholtz \& Hall 2016). Diese Erfahrungsart ist unmittelbarer Natur, ein Individuum erlebt direkt. Beide Erfahrungsarten sind grundlegend auf Sprache aufgebaut. Wenngleich die vermittelte Erfahrung auch ikonisch geschehen $\mathrm{kann}^{10}$, ist die direkte Erfahrung mindestens im Reflexionsprozess immer sprachlich-symbolisch. Schmerz, beispielsweise, ist zunächst als Feuern von Neuronen physikalisch beschreibbar. Die Sprache wird relevant, wenn über die Ursache, die Intensität oder die Folgen des Schmerzes reflektiert wird. Dass dabei des Öfteren metaphorisch und metonymisch gespro-

2014: 64-94) wie die Modularitätshypothese, die jeglicher Evidenz entbehrt (vgl. Evans 2014: 133-160).

10 Auch in vermittelten Erfahrungsprozessen spielt Sprache zumeist eine zentrale Rolle, da über die Erfahrungsobjekte gesprochen wird und so mehr Information zugänglich gemacht werden kann als rein über den visuellen Reiz. 
chen wird, erschwert die Entschlüsselung des Sinns, vereinfacht im Gegenzug aber die Konzeptualisierung.

In den alltäglichen Lebenswelten spielt die Reflexion über Sprache selbst eine entscheidende Rolle; sie stellt die Grundlage aller Konzeptualisierungen von Sprache dar (vgl. Spitzmüller 2019). ${ }^{11}$ Die Sprachreflexivität ist deshalb so zentral, da der Mensch ${ }^{12}$ im Rahmen einer „cognitive liberation and human transcendence“ (Coupland \& Jaworski 2004: 17) sein eigenes Dasein überschreitet und vom bloßen Teilnehmer zum Beobachter wird. ,Der Alltag‘ kann infolgedessen als „Stätte kontingenter Sinnbildung, Sinnverteilung und Sinnablagerung und Stätte von Sinnentstellungen und Sinnverdrängungen, von Schein und Gewalt, und all dies in labyrinthischer Mischung“ (Waldenfels 1985: 176) verstanden werden. Die Emphase liegt dabei vor allem auf dem Alltag als „Stätte kontingenter Sinnbildung““. ${ }^{13}$ Die Sinnbildung soll allerdings nicht den Eindruck vermitteln, alle Reflexionsprozesse liefen bewusst ab. Vielmehr begegnet der Mensch seiner Umwelt zumeist in einer „natürlichen Einstellung“ (Husserl 1976: 57), d. h. in bloßer Akzeptanz zu den ihn umgebenden Zuständen, die ohne Anlass keiner eingehenderen Reflexion unterzogen werden.

\subsection{Charakteristika von (Meta-)Sprachwissen in alltäglichen Lebenswelten}

Das Transzendieren der Weltgrenzen (vgl. Wittgenstein 1921 / 2016: 67) bzw. die Fähigkeit zur Metasprache überhaupt setzt dabei ein Wissen von der Welt voraus, das als holistisches Konzept Sprachwissen inkludiert. ${ }^{14}$ Dieses Sprachwissen kann einerseits aus einer diskurstheoretischen und andererseits aus einer kognitionstheoretischen Perspektive beschrieben werden. Die linguistischen Disziplinen, die sich - mal mehr, mal weniger explizit - mit Wissen auseinandersetzen, sind zahlreich. Die Bandbreite reicht von Diskurslinguistik (vgl. z. B. Spitzmüller \& Warnke 2011) zu (Konstruktions-)Grammatik (vgl. z. B. Stefanowitsch 2011) über Pragmatik (vgl. z. B. Deppermann 2015) und (Historischer) Semantik (vgl. z. B. Busse 1987) bis zur Variationslinguistik (vgl. z. B. Purschke

11 Eine für diesen Beitrag fruchtbringende Synthese von alltäglichen Lebenswelten und kognitiver Linguistik liefert Konerding (2001).

12 Es geht also nicht um die Unterscheidung von Objekt- und Metasprache, sondern um die sog. ,folk metalanguage“ (vgl. Preston 2004).

13 Vgl. dazu auch Felder \& Gardt (2018) sowie Searle $(1997,2012)$.

14 Dieses Wissen kann im Anschluss an die natürliche Einstellung (vgl. Abschnitt 2.2) mit Polanyi (1985) als implizites Wissen gelten. 
2011). Auch diesen Disziplinen liegt gewissermaßen die dichotome Trennung in diskurs- und kognitionstheoretische Ansätze zugrunde, wenngleich dies nicht immer expliziert wird (vgl. z. B. Warnke 2018 für die Diskurslinguistik).

Bisher haben sich in den verschiedenen Ausprägungen der Laienlinguistik vor allem Ex-negativo-Beschreibungsansätze für das Laienwissen durchgesetzt, die Laienwissen als vom Expertenwissen abweichend konzeptualisieren (vgl. Spitzmüller in diesem Band). Diese Gegenüberstellung, die sich in der Dichotomie von Laien- und Expertentheorien noch verstärkt, hat ein grundlegendes Problem der Vergleichbarkeit. In Erhebungssituationen wird Wissen bei Laien i. d. R. ad hoc generiert und abgefragt, während das Wissen der Experten als Vergleichsgrundlage zumeist auf Basis von Veröffentlichungen (oder anderen textuellen Grundlagen) generiert wird. Insofern ist Laien- und Expertenwissen schon aus methodologischer Perspektive nicht vergleichbar, wenn die Erhebungssettings nicht ähnlich sind (Validität). Einen empirischen Nachweis über die strukturelle ,Überlegenheit‘ des Expertenwissens ist die Forschung bisher schuldig geblieben. Angenommen werden kann allerdings eine durch divergierende gesellschaftliche Positionen (insbesondere im Rahmen von Institutionalisierung) bedingte ,Andersartigkeit‘ des Expertenwissens im Hinblick auf Inhalte, Distribution und Funktion (vgl. Antos 1996: 29). Da der Ansatz, Laienwissen als in bestimmter Weise ,strukturanalog' (vgl. Furnham 1988: 2) zu Expertenwissen $\mathrm{zu}$ begreifen, bisweilen eine gewisse Konjunktur erlebt, überrascht es doch, wenn in der Forschung Laien immer noch als defizitär gegenüber dem Experten konzeptualisiert werden. Ihr Wissen sei nach Antos (1996: 29-34) situationsbezogen, wertbezogen, affektbezogen, implizit, lückenhaft, inkohärent, inkonsistent sowie falsifikationsresistent. ${ }^{15}$ Insbesondere in Bezug auf die Lückenhaftigkeit stellt sich die Frage, ob diese Eigenschaft dem Expertenwissen als Spezialwissen nicht in übergeordnetem Maße ebenfalls zukommt und zukommen muss. Die Expertise (vgl. Kasper \& Purschke in diesem Band) zeichnet sich eben gerade durch Lückenhaftigkeit in einigen Bereichen und der dadurch bedingten Möglichkeit der intensiven Beschäftigung mit anderen Bereichen aus. Experten können also immer nur dann Experten sein, wenn sie in Kauf nehmen, Laien in anderen Bereichen zu sein und wenn ihre Expertise akzeptiert, autorisiert und legitimiert wird. ${ }^{16}$

15 Vgl. dazu auch Hundt (2017: 139), der, wenngleich mit anderen Termini, ähnliche Konzepte vertritt.

16 Universalgelehrsamkeit sei in Anbetracht einer sich immer stärker differenzierenden und beschleunigenden Welt an dieser Stelle ausgeklammert und soll als historische Angelegenheit verstanden werden. 
Eine neutral ausgerichtete Beschreibung der Struktur laienlinguistischen Wissens kann als „geteilte Akzeptanz von Erkenntnis“ (Warnke 2009: 113) erfolgen. ${ }^{17}$ Diese diskurslinguistische Sichtweise bezieht sich auf „kollektive Meinungen und zugehörige sprachliche Darstellungen, die als unstrittig geteilt werden und über deren Akzeptanz ein relativer Konsens besteht“ (Konerding 2015: 60). In dieser situativen Varianz von intersubjektiv als gültig anerkannten Aussagen als Wissen geht es immer um individuell relevante „kognitive Ordnungsschema[ta], [denen] ein bestimmtes Kollektiv in einer gegebenen historischen und kulturellen Situation ontologischen Status zuspricht“ (Spitzmüller 2009: 114, Herv. i. Orig.). Diese Zuweisung von ontologischem Status in je bestimmten Situationen bedeutet nicht, dass das Wissen zufälligen Charakter hat, variabel und orientierungslos ist, ganz im Gegenteil: Die Wissensbestände bilden die Basis für ihrerseits „kohärente“ (Spitzmüller 2009: 188) Sprachtheorien, die sich nicht durch Widerspruchsfreiheit auszeichnen müssen, sondern „auf individuellem Erfahrungswissen [vermittelt oder unmittelbar, s. o., T. H.] basieren und sich so [...] bilden und verfestigen“ (Hoffmeister 2019: 169). Wenn Hoffmeister (2019: 169) davon ausgeht, dass sich die Theorien „ohne den Einfluss Dritter bilden und verfestigen“, so referiert dies nicht auf den Input des Wissens, der ohne soziales Moment ohnehin nicht denkbar ist, sondern es bezieht sich vielmehr auf die individuelle kognitiv-prozessuale Verarbeitung des Wissens (vgl. Hoffmeister 2020a).

Für die Laienlinguistik muss also ein konsensualistisch-orientierter Wissens- und Wahrheitsbegriff angenommen werden (vgl. Habermas 1973, Westerkamp in diesem Band), der aber dennoch die jeweiligen kognitiven Realitäten berücksichtigt (vgl. Hoffmeister 2020a). Aus diesem Grund soll im Folgenden eine kognitive Laienlinguistik skizziert werden, die diskursive Aspekte als Basis der Sozialität des Wissens anerkennt, aber die individuell-kognitive Ebene fokussiert.

\section{Umrisse einer kognitiven Laienlinguistik}

Eine kognitive Laienlinguistik erspart sich die Probleme, die in Abschnitt 2.3 in Bezug auf die Ex-negativo-Konstruktion des Laien beschrieben wurden, da sie

17 Mit den Worten Jürgen Links (2005: 78) könnte man sagen, es herrsche ein „interpersonaler Aktionismus“ vor, der schließlich in einen „Elementardiskurs“ (Link 2005: 79) münde. 
den Laien nicht als dichotom ${ }^{18} \mathrm{zu}$ Experten versteht, sondern seine Wissensbestände individualistisch, wenngleich nicht ohne Berücksichtigung sozialer Dimensionen, untersucht. Es wird also die Repräsentation sprachlichen Wissens auf Basis individueller Voraussetzungen untersucht. Gleichwohl ist eine gewisse Sozialität des Wissens nicht zu vernachlässigen, sodass es sich tatsächlich eher um eine Form der sozio-kognitiven Laienlinguistik (vgl. Cuonz 2014) handelt.

Im Folgenden sollen nun die Prinzipien der kognitionslinguistisch ausgerichteten Laienlinguistik dargestellt werden. ${ }^{19}$ Danach wird im Anschluss an Hoffmeister (2020c) die Theorie der Repräsentation von Metasprachwissen im Rahmen eines Epistemikons (als Kofferwort von altgriech. episteme [,Wissen'] und Lexikon) erläutert.

\subsection{Die fünf Basisprinzipien der kognitiven Laienlinguistik}

Das symbolische Prinzip (1) ${ }^{20}$ verweist auf den Symbolcharakter des Wissens. Dabei geht es darum, dass die einzelnen Wissenselemente (Episteme) einerseits eine Inhaltsseite und andererseits eine (oder auch mehrere verschiedene) Ausdrucksseiten $^{21}$ besitzen; es handelt sich also um ,symbolische Einheiten' (vgl. Ziem 2009: 175). Die Inhalts- und Ausdrucksseiten sind dabei untrennbar miteinander verbunden, besitzen konventionellen Charakter, sind aber dahingehend arbiträr, dass das jeweilige Individuum für die Wahl einer perspektivierenden Ausdrucksseite verantwortlich ist. Damit erfüllt die Ausdrucksseite eine Indikatorenfunktion für die jeweilige Perspektivierungsweise des Wissens, die das Individuum durch die Wahl einer bestimmten Ausdrucksseite intendiert.

Wenn ein Epistem durch unterschiedliche Ausdrucksseiten repräsentiert wird, so wirken die unterschiedlichen Ausdrucksseiten je perspektivierend,

18 Eine dichotome Sichtweise birgt indes an sich schon eine gewisse Problematik, da eine Kompetenzzuschreibung eher graduell zu denken ist: Jemand ist mehr oder weniger ein Laie / Experte (vgl. Hoffmeister 2019: 166).

19 Für die Kognitionslinguistik wurden die Prinzipien ausführlich von Ziem (2009: 175-176, 2013) beschrieben. Diese Prinzipien werden hier auf die Laienlinguistik angewendet.

20 Vgl. dazu grundlegend Ziem (2008: 177-180).

21 Dass das Wissen eine Ausdrucksseite besitzt, ist weniger strukturell zu verstehen als es der Saussure'sche Begriff impliziert. In der Forschungspraxis zeigt sich des Öfteren, dass einzelne Wissensbestände nicht oder nur unzureichend expliziert werden können (vgl. Hundt 2017: 140; Ziem 2014: 16). Die Ausdrucksseite beschreibt also eher ein Ausdruckspotential, bei dem einzelne Episteme ungeäußert bleiben können (vgl. Antos 1996: 31). 
sodass durch die jeweilige „Wahrnehmungsperspektive konkrete Vorstellungsinhalte für einen Adressaten objektiviert werden“ (Köller 2004: 21). Erkennbar ist dieser Umstand in gegenwärtigen Debatten zur sog. gendergerechten Sprache, die in bestimmten Kontexten u. a. auch als ,Gendern', ,Gender-Sprache', ,Genderwahn', ,Gender-Unfug' oder ,Gendergaga' bezeichnet wird. ${ }^{22}$ Dabei wird deutlich, dass bei den unterschiedlichen, perspektivierenden Ausdrucksseiten Wissen repräsentiert wird, das neben der rein kognitiven auch eine konative, d. h. handlungsleitende sowie eine emotive Komponente eine Rolle spielt (vgl. Haddock \& Maio 2014; Schmidlin 2011: 181-194).

Dass Wissen einerseits Resultat von (institutionalisierten) Vermittlungsprozessen im Rahmen von taught experience und andererseits von direct experience sein kann, wird durch das sog. Erfahrungsprinzip (2) beschrieben, das in Abschnitt 2.2 bereits näher erläutert wurde.

Das Resultat sind schließlich diverse Konzepte (Konzeptualisierungsprinzip (3)), die sich in ihrem Komplexitätsgrad unterscheiden. Insofern ist (meta)sprachliches Wissen „vielmehr ein Epiphänomen, das genauso konzeptuellen Prozessen unterworfen ist wie jede andere Wissensform auch“ (Ziem 2008: 178).

Das auf die eine oder andere Art erworbene Wissen wird, so (4) das Kategorisierungsprinzip, kognitiv kategorisiert, $\mathrm{d}$. h. die Episteme werden entweder auf der Type-Ebene abstrahiert und anschließend geclustert oder direkt auf der Token-Ebene kategorisiert, z. B. wenn eine konkrete Äußerung in der Sprachrezeption und -perzeption einer bestimmten Kategorie zugeordnet wird (Konzeptcluster als Frames, vgl. Busse 2012: 540-542). Dieser Vorgang ist essentiell, um Wissen in neuen, unbekannten Situationen schneller abrufen und anwenden zu können. Damit einher geht z. B. auch die Stereotypenbildung sowie die Bewertung von Einheiten hinsichtlich von Prototypizitätsgraden.

Frames als Strukturen, die aus Konzepten bestehen, sind schließlich die Grundeinheiten jeglichen Wissens, „das Format schlechthin der Strukturen des Wissens“" (Busse 2012: 539, Herv. i. Orig.). ${ }^{23}$

Schließlich beschreibt (5) das Gebrauchsbasiertheitsprinzip, dass die einzelnen Episteme am Gebrauch orientiert sind. Die Konzepte entstehen also aus der

22 Die Bezeichnungsalternativen sind schier unüberblickbar, die Auflistung erhebt keinen Anspruch auf Vollständigkeit. Für einen linguistischen Blick auf den Gegenstand vgl. AlkerWindbichler (in diesem Band) sowie Kotthoff \& Nübling (2018).

23 „In addition to seeing frames as organizers of experience and tools for understanding, we must also see frames as tools for the description and explanation of lexical and grammatical meaning“" (Fillmore 1985: 232). 
Reflexion des Sprachgebrauchs ${ }^{24}$ und sind in der Verarbeitung Resultate von Entrenchmentprozessen sowie kognitiver Routinen. In Bezug auf dieses Prinzip lässt sich eine zweifache Gebrauchsbasiertheit feststellen, da das Wissen einerseits aus dem Sprachgebrauch emergiert, d. h. sprachgebrauchsgebunden und diskursiviert ist und andererseits sprachgebrauchsbezogen ist, d. h. das Sprechen selbst reflektiert. Die semantischen Strukturen sind „zugleich als Resultate des kommunikativen Gebrauchs von sprachlichen Ausdrücken und als sedimentierte[r] Wissensbestand, über den SprachbenutzerInnen verfügen“ (Ziem 2013: 219) zu verstehen. Dieser Umstand betont die Sozio-Kognitivität des Metasprachwissens.

Aus den eben dargestellten Überlegungen zu den fünf Basisprinzipien der kognitiven Laienlinguistik folgt, dass (Meta)Sprachwissen in diesem Kontext als ein holistisches Modell (vgl. Ziem 2008: 117-172) verstanden werden muss. Modulare Konzepte wie die der generativen Grammatik sind für diesen Ansatz also nicht tragfähig (vgl. Fußnote 9).

\subsection{Wissen als Semantik im Epistemikon}

Die Theorie der Repräsentation von Wissen als Semantik im Epistemikon ${ }^{25}$ schließt unmittelbar an die in Abschnitt 3.1 dargestellten Basisprinzipien der kognitiven Laienlinguistik an.

Als Epistemikon wird dabei das Netzwerk aller kognitiv repräsentierten Sprachwissensbestände bezeichnet (grammatisches, lexikalisches etc. Wissen aber auch Metasprachwissen). Es erhebt den Anspruch eine Form der ,MetaTheorie' zu sein, d. h., dass bspw. das Konstruktikon der Konstruktionsgrammatiken ein Bestandteil des Epistemikons ist und auch die Konstruktionen im Epistemikon repräsentiert sein können. Der Fokus in der vorliegenden Arbeit liegt allerdings in der Funktion des Epistemikons, Metasprachwissen theoriegebunden zu erklären, das Wissen wird als Semantik konzeptualisiert, sodass das Epistemikon stets eine inhaltsorientierte Perspektive einnimmt. Die einzelnen Episteme (,semantische Einheiten') ${ }^{26}$ unterhalten dabei „systematische Bezie-

24 Vgl. dazu auch das ,Modell metapragmatischer Positionierung، von Spitzmüller (2013: 273). 25 Die Darstellung der Theorie soll hier in aller gebotenen Kürze erfolgen. Ausführlichere Darstellungen finden sich in Hoffmeister (2020c).

26 Episteme können mit Busse (2018: 8) definiert werden als „menschliches Wissen, gleich welcher Art, Herkunft und Entstehungsform“, es sind die Wissensbestandteile im Epistemikon, die als semantische Einheiten eine Inhaltsseite und mindestens eine Ausdrucksseite besitzen. 
hungen untereinander“, die sie „so erst zu Wissen im Sinne von kognitiv verfügbaren und anwendbaren Einheiten - wenngleich nicht zwangsläufig explizierbaren - Einheiten des Langzeitgedächtnisses werden lassen“ (Ziem 2014: 16). Die Nicht-Explizierbarkeit des Wissens (s. o.) ist ein zentraler Gedanke, da es in Erhebungssituationen oftmals vorkommt, dass Wissen zwar vorhanden ist, aber nicht näher erläutert werden kann, z. B. weshalb der sächsische Dialekt als ,hässlich` empfunden wird oder warum es falsch ist, ,Peter ist größer als wie seine Schwester' zu sagen. Das Epistemikon ist also zuvorderst ein „strukturiertes Inventar von Wissensbeständen“ (Ziem \& Lasch 2013: 2). Die einzelnen Episteme sind mindestens bilateral und potentiell multilateral strukturiert (vgl. das symbolische Prinzip, Abschnitt 3.1 sowie Langacker 1987: 77). Interindividuell aber auch intraindividuell sind die Wissensbestände unterschiedlich komplex und abstrakt. Der jeweilige Komplexitätsgrad hängt dabei von vielfältigen Faktoren ab, z. B. vom jeweiligen Vorwissen aber auch vom Interesse und von motivationalen Aspekten. Insofern wird bei der Aktivierung von einzelnen Epistemen dieses Vorwissen (der Kontext bzw. ein spezifischer Frame) immer mit aktiviert $^{27}$, sodass sich für das Epistemikon festhalten lässt, dass es sich um ein größtenteils aus Erfahrung gespeistes „Netzwerk von Assoziationen [und Perzeptionen, T. H.]“ (Goldberg 1995: 5, Übers. nach Ziem \& Lasch 2013: 11) handelt. Die einzelnen Sprachkonzepte, die sich zu Frames clustern, werden semantisch durch Assoziationen und Perzeptionen, d. h. Episteme, , gefüllt ${ }^{\star 28}$, die wiederum miteinander verknüpft sind. So entsteht das mehr oder weniger komplexe Netzwerk aus Assoziationen und Perzeptionen (,semantische Einheiten'), in dem potentiell alles miteinander verbunden sein kann. Diese Verbindungen offen zu legen, ist ein Ziel laienlinguistischer Forschung. Der Theorie liegt ein Verständnis des Laienwissens als ,subjektive Theorie‘ zugrunde (vgl.

Diaz-Bone (2013) spricht von Sozioepistemen und trägt damit der Sozialität Rechnung. Im vorliegenden Beitrag wird allerdings der allgemeinere Begriff Epistem verwendet.

27 Die Verbindung von Epistemen und Frames muss im Rahmen weiterer Studien näher untersucht werden. An dieser Stelle genügt folgende Feststellung: Episteme haben prinzipiell eine andere Perspektive auf sprachliches Wissen als Frames, da sie keine Leerstellen produzieren, sondern mehr oder weniger feste Verbindungen aus Form und Bedeutung darstellen. Allerdings werden die Episteme in entsprechende Frames eingebettet, sodass ein Zusammenhang besteht. Insofern gibt es Fälle, in denen Episteme als konkrete Realisierung von Konzepten verstanden werden können. Grundsätzlich stellen Sie aber generelle, formbedeutungsbasierte Wissenseinheiten dar, d. h., dass auch Konzepte und sogar Frames Episteme sind.

28 ,Gefüllt‘ werden sie deshalb, da die Struktur eines Frames Slots (,Leerstellen') und Filler (,Füllwerte`) vorsieht, die die Semantik näher explizieren (vgl. Busse 2012: 553-594). 
Hoffmeister 2020b), wenngleich der Terminus ,Theorie‘, wie oben beschrieben, nicht unproblematisch ist. Der Ansatz wird allerdings dadurch legitimiert, da schon Fillmore (1982: 111) den Frame-Begriff als Hyperonym u. a. zu „folk theories" verwendet. ${ }^{29}$

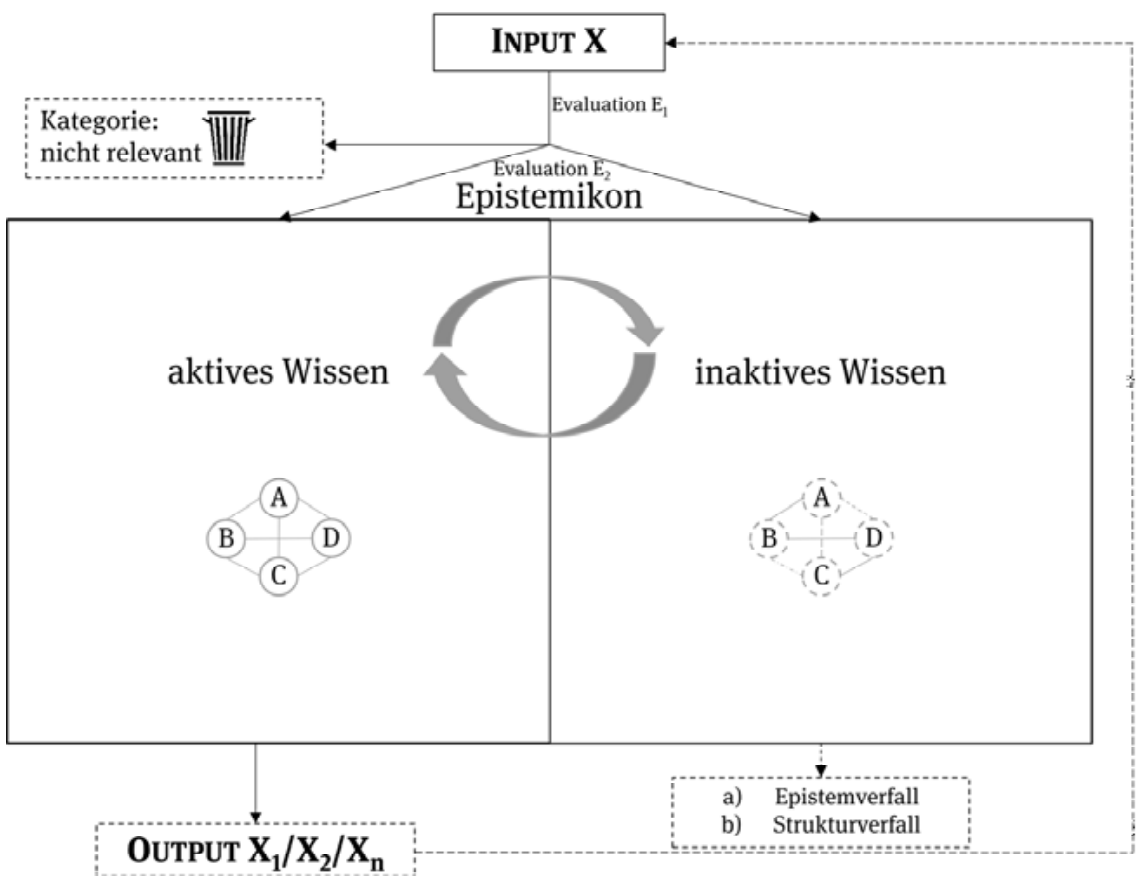

Abb. 1: Entstehung und Struktur des Epistemikons, revidiert (nach Hoffmeister 2020a).

In Abb. 1 ist die Entstehung und die Struktur des Epistemikons im Anschluss an Hoffmeister (2020c) dargestellt. Der Input stellt dabei entweder eine taught oder eine direct experience dar, die als potentielles Epistem einer Evaluation $\left(\mathrm{E}_{1}\right)$ unterzogen wird. ${ }^{30}$ Wenn der Input als nicht-relevant beurteilt wird, findet keine

29 „I intend the word ,frame“ as used here to be a general cover term for the set of concepts variously known, in the literature on natural language understanding, as ,schema', ,script", ,scenario', ,ideational scaffolding', , cognitive model“ or ,folk theory““ (Fillmore 1982: 111).

30 Dieser Gedanke schließt an die Ausführungen von Schütz \& Luckmann (2003) an. Dabei wird der Gedanke übernommen, dass ein gesellschaftlicher Wissensvorrat seinen Ursprung in der Subjektivität allen Wissens haben muss (vgl. Schütz \& Luckmann 2003: 355-356, weiterführend Schröder 2019: 37-40). Ergänzt wird dieser Ansatz im vorliegenden Verständnis um 
nähere Reflexion statt. Wird die Erfahrung allerdings als relevant beurteilt, so erhält sie Eingang in das Epistemikon und wird dort erneut evaluiert $\left(E_{2}\right)$. Weicht $\mathrm{E}_{2}$ von $\mathrm{E}_{1}$ qualitativ ab, so wird das Ergebnis der zweiten Evaluation der ersten vorgezogen. Anschließend wird das Epistem je nach Relevanz in die Kategorien aktives Wissen (explizierbar) und inaktives Wissen (nicht explizierbar) unterteilt. Im Rahmen von Re-Evaluationen wird der Status jedes Epistems überprüft, sodass bei mangelnden Aktivierungsreizen ein aktives Wissen zu inaktivem werden kann bzw. inaktives Wissen aktiviert und so zu aktivem Wissen werden kann. Einzig das aktive Wissen ist outputfähig. ${ }^{31}$ Dieser Output kann (normalerweise durch taught experience) schließlich in anderen Situationen erneut zum Input werden. I. d. R. weicht er von dem ursprünglichen Input aufgrund der verschiedenen Reflexionsprozesse ab. Das inaktive Wissen kann potentiell vergessen werden, zwei verschiedene Vorgänge sind dabei denkbar: Es kann a) zu einem Epistemverfall kommen, bei dem Wissensinhalte, also die Episteme, nicht mehr (ausreichend) erinnert werden können. Dies könnte als eine Form des ,Detrenchments“ verstanden werden. Darüber hinaus kann es b) $\mathrm{zu}$ einem Strukturverfall kommen. Dieser betrifft die einzelnen Kategorisierungslinks der Frames, die nicht mehr stabil sind und zu unfesten bzw. fehlenden Verbindungen zwischen den einzelnen Konzepten führen, was dazu führt, dass das Epistem nicht mehr oder nur noch über einen anderen Input aktiviert werden kann.

Innerhalb des Epistemikons sind die einzelnen Konzepte zu Framestrukturen mehr oder weniger gefestigt (vgl. die exemplarische ABCD-Darstellung, Abb.1). Dabei sind potentiell alle Konzepte miteinander verknüpft. Allerdings können im inaktiven Wissen einige Verbindungen fester als andere sein, wenngleich sie insgesamt per se weniger fest sind, als im aktiven Teil. Die Dynamik nimmt mit dem Grad der Inaktivität $\mathrm{zu}$.

Die Theorie des Epistemikons ist schließlich der Ausgangspunkt für die empirische Rekonstruktion der in der Öffentlichkeit vorhandenen Sprachkonzepte.

eine kognitivistische Sicht auf den ,subjektiven Wissensvorrat', sodass das Resultat ein soziokognitiver Ansatz ist.

31 Für anschließende Studien wären folgende Fragen untersuchenswert: Wie wird Wissen überhaupt aktiviert (vgl. Hoffmeister 2020a)? Welche Parameter bestimmen die Aktivierung von Wissen und, ob Wissen überhaupt outputfähig ist (vgl. zum Priming exemplarisch Palliwoda 2019)? 


\section{Das Konzept Deutsche Sprache in der Öffentlichkeit}

In Abschnitt 4 wird ein Ausschnitt einer Erhebung vorgestellt, die zum Ziel hat, die einzelnen Sprachkonzepte in der Öffentlichkeit abzubilden. ${ }^{32}$ Dabei werden im vorliegenden Beitrag drei Gewährspersonen (GP) exemplarisch analysiert, um die Struktur der Studie vorzustellen. Die Ergebnisse (Abschnitt 4.3) sind also nicht verallgemeinerbar und haben bloß zum Ziel, die empirische Anwendbarkeit der Theorie in Abschnitt 3.2 zu testen. ${ }^{33}$

\subsection{Ausgangsfragen, Hypothesen und das Ziel der Untersuchung}

Im folgenden Abschnitt steht die Frage im Vordergrund, wie linguistische Laien die deutsche Sprache konzeptualisieren. Das Ziel ist also, ein Konzept DEUTSCHE SPRACHE aus einer synchronen Perspektive zu rekonstruieren. ${ }^{34}$ Die Basis sind qualitative Daten, d. h. es geht um lexikalisch gebundenes Bedeutungswissen (vgl. Ziem 2020).

Das zweite, langfristige Ziel sollte dabei die Untersuchung gesellschaftlich gefestigter Sprachkonzepte (sog. default values, ,Standardwerte') ${ }^{35}$ sein. Im Rahmen des vorliegenden Beitrages liegt der Fokus allerdings, bedingt durch die kleine Stichprobe, auf individueller Ebene. Im Zuge dessen lässt sich vermuten, dass die Konzepte zwar standardisiert sind, sich aber durch eine sog. FillerVarianz unterscheiden (H1: Standardisierungshypothese). Außerdem unterscheiden sich die GP mutmaßlich stark in Bezug auf die Komplexität (H2: Komplexitätshypothese). Gründe für die Komplexitätsheterogenität könnten vor allem in der persönlichen Biografie (z. B. durch sprachaffine Berufe) sowie im Bildungsgrad liegen. $\mathrm{Zu}$ vermuten ist des Weiteren, dass die Konzeptassoziationen aufgrund des Stimulus (vgl. Abschnitt 4.2) i. d. R. nominal repräsentiert

32 Für eine umfassende Darstellung der Ergebnisse mit einer größeren Stichprobe vgl. Hoffmeister (2020c).

33 Für eine repräsentative Erhebung von Spracheinstellungen vgl. Adler \& Plewnia (in diesem Band) sowie ergänzend zu diesem Beitrag Klein (in diesem Band), der den Fokus auf „die deutsche Grammatik“ legt.

34 Vgl. dazu auch Sauer (in diesem Band), die Spracheinstellungen aus diachroner Perspektive framesemantisch abbildet.

35 Zum Verhältnis von Epistemikon und Framesemantik vgl. Abschnitt 3.2. 
sind und somit auch auf diese Weise sprachlich wiedergegeben werden (H3: Repräsentationshypothese). Der Grund dafür ist die Reiz-ReaktionsKompatibilität (vgl. Proctor \& Vu 2006). Allerdings ist aufgrund der Erfahrungsvariation nicht davon auszugehen, dass Reaktionen bzw. Assoziationen wie in der Vorstellung des Reiz-Reaktions-Modells (vgl. Skinner 1957) interindividuell vergleichbar sind (vgl. Hörmann 1978: 28-32), da die Reaktionen hier nicht, wie im klassischen Behaviorismus postuliert, aus den Reizen (Stimuli) ableit- und damit vorhersagbar sind.

Insgesamt kann das Ziel der Untersuchung als ein erster Versuch zusammengefasst werden, einen Überblick über die „kodifizierte Alltagstheorie der Sprache“ (Antos 1996: 26) zu erhalten. ${ }^{36}$

Im Folgenden sollen nach der Darstellung des Untersuchungssettings die Hypothesen anhand von drei ausgewählten GP exemplarisch beleuchtet werden.

\subsection{Das Untersuchungssetting und die Stichprobe}

Die Untersuchung basiert auf einem Wortassoziationstest in der Tradition von Carl Gustav Jung (1979). Das Vorgehen wird allerdings dahingehend verändert, dass nicht mehrere Stimuli präsentiert werden, auf die anschließend je eine Reaktion erfolgt, sondern es wird ein einziger (nominaler) Stimulus (,Deutsche Sprache') präsentiert, auf den die GP im Anschluss reagieren. In einem zweiten Interviewteil werden die einzelnen Items mit dem Ziel abgefragt, nähere Erklärungen zu erhalten. Neu aufgenommene Konzepte der GP werden ihrerseits erneut abgefragt. ${ }^{37}$ So entsteht ein umfassenderes Bild der Konzeptualisierungen zur deutschen Sprache.

Zwar haben die Reaktionen der GP den Charakter von Assoziationen, es wird mit Jung (1979) allerdings der Terminus ,sprachliche Reaktion' präferiert, da es sich um lexikalisch gebundenes Wissen und damit um eine Reflexion handelt und weniger um nicht explizierte kognitive Verknüpfungen.

36 ,Kodifiziert“ bezieht sich bei Antos (1996: 35) auf die Festlegung der Theoriebestandteile in den von ihm untersuchten Sprachratgebern. In dem vorliegenden Beitrag bezieht sich das Attribut eher auf die Hermetik, d. h. die Abgeschlossenheit gegen äußere Einflüsse bzw. die Existenz von default values und ist damit eher metaphorisch zu verstehen.

37 Nun ist es sicherlich nicht unproblematisch, wenn der Explorator in der laufenden Interviewsituation ein Urteil darüber fällt, was als neues Konzept zu gelten hat (und was nicht), allerdings lässt sich die Einschätzung durch die Neuartigkeit des Punktes im Interview sicherlich entsprechend plausibilisieren. 
Die Grundannahme ist, dass die Vorstellungen miteinander verknüpft sind und deshalb auch so sprachlich repräsentiert werden. Dies schließt an die holistische Betrachtung der kognitiven Semantik an (kognitiv-semantische Netze).

Tab. 1: Soziodemographie der Untersuchungsstichprobe.

\begin{tabular}{|c|c|c|c|}
\hline GP & Sexus & Geburtsjahr & Ausbildung \\
\hline 01 & M & 1977 & $\begin{array}{l}\text { Schiffbauingenieur/Polizeiausbildung/Feuerwehrausbildung } \\
\text { (aktuell) }\end{array}$ \\
\hline 02 & M & 1958 & $\begin{array}{l}\text { Lehrer für Deutsch und Musik/Geschäftsführer Wohlfahrtsverband } \\
\text { (aktuell) }\end{array}$ \\
\hline 03 & W & 1931 & Büroangestellte/Sekretärin (Ruhestand) \\
\hline
\end{tabular}

Die Stichprobe besteht aus drei GP (zwei männliche und eine weibliche GP). Sowohl bei dem Geburts- wie auch dem Wohnort wurde auf Homogenität geachtet. Die Bedingung dabei war, dass die GP aus Orten nördlich der Benrather Linie stammen, um eine aktive Dialektkompetenz mittel- und oberdeutscher Dialekte auszuschließen. ${ }^{38}$ In Tab. 1 sind die soziodemographischen Daten (Sexus, Geburtsjahr und Ausbildung) der GP, die für die Auswertung relevant sind, zusammengefasst.

\subsection{Konzeptualisierungen in den einzelnen Epistemika}

Im Folgenden werden die Ergebnisse dargestellt. Drei Hauptaspekte konnten für die vorliegenden Daten als besonders relevant ermittelt werden. Dabei handelt es sich um die Konzepte VARIATIOn, Norm bzw. STANDARDiSIERUnG sowie TRADITION, die zunächst inhaltlich beschrieben (Abschnitte 4.3.1 bis 4.3.4) und anschließend (Abschnitt 4.4) auf die in Abschnitt 4.1 entwickelten Hypothesen hin überprüft werden. Im Folgenden findet sich jeweils eine Darstellung des Epistemikons der jeweiligen GP. In den Abbildungen 2-4 finden sich die Dar-

38 Dass Dialektkompetenz bzw. die regionale Herkunft einen Einfluss auf das Dialektwissen haben können und damit auch auf die Konzeptualisierungsleistung, hat bspw. Hoffmeister (2017) zeigen können. Aus diesem Grund ist eine größtmögliche Homogenität zentral. 
stellungen der Epistemika. ${ }^{39}$ Auf weitere Aspekte, die neben den oben genannten eine Rolle spielen, kann an dieser Stelle leider nicht eingegangen werden.

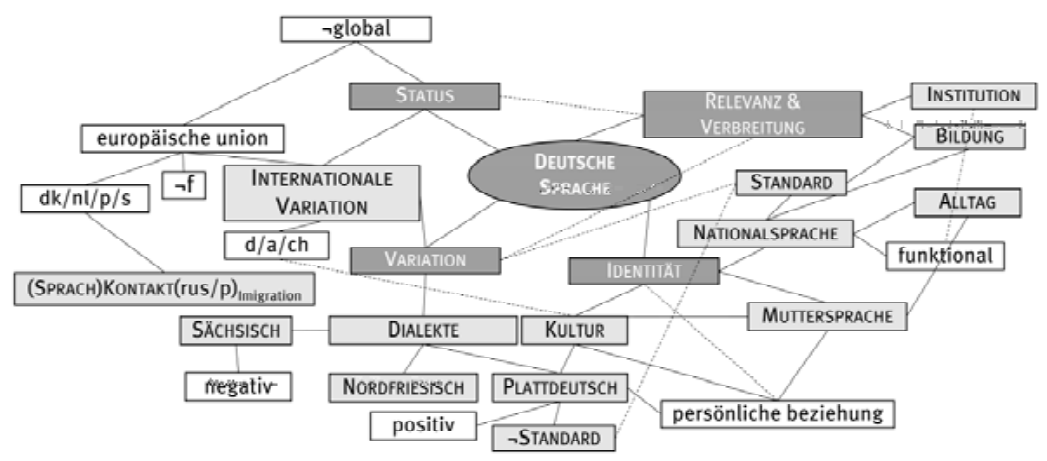

Abb. 2: Darstellung des Epistemikons von GP 01.

39 Die Darstellung orientiert sich in ihrer Struktur an der Darstellung des Konstruktikons in Lasch (2016: 514-515). Einige Bemerkungen müssen zu den Abbildungskonventionen gemacht werden: Grau hinterlegt (weiße Schrift, Kapitälchen) ist die Makroebene, auf der Basiskonzepte verortet werden. Hellgrau hinterlegt sind untergeordnete Konzepte (Mesoebene). Weiß und mit Minuskeln sind relevante Beispiele oder Konzept- ergänzungen ausgezeichnet. Eine Unterscheidung in Bezug auf die Festigkeit der Verbindungen der Konzepte kann leider nicht getroffen werden. Linien zeigen diese Verbindungen zwischen den einzelnen Konzepten auf, die von der GP selbst hergestellt wurden. Die gestrichelten Linien verweisen auf Verbindungen, die zwar eine kognitive Realität besitzen, von den GP selbst aber nicht thematisiert werden. Dies stellt eine Interpretationsleistung des Forschers dar (vgl. Abb. 2-4). 


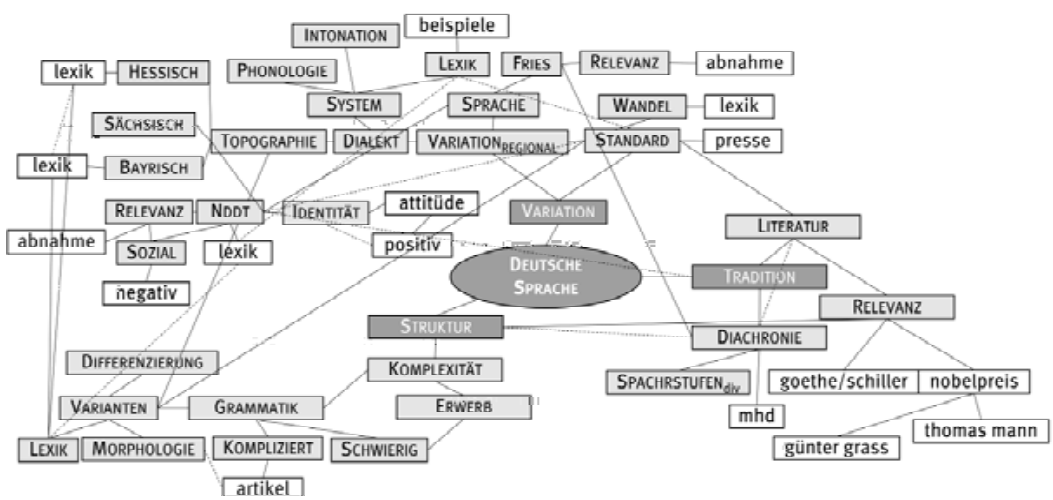

Abb. 3: Darstellung des Epistemikons von GP 02.

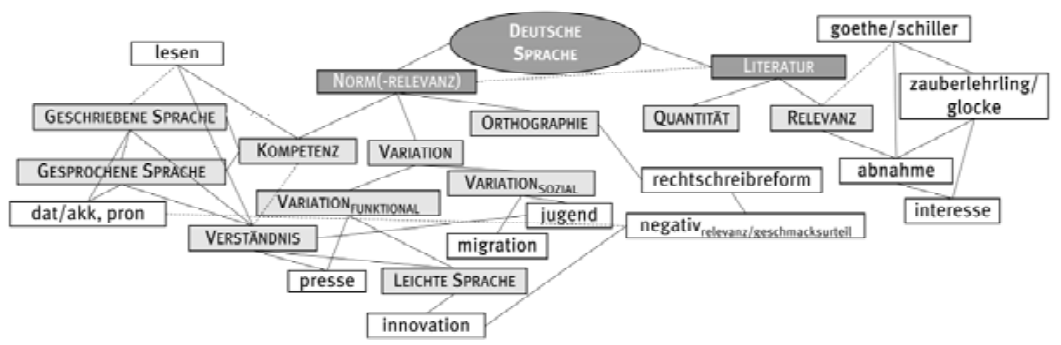

Abb. 4: Darstellung des Epistemikons von GP 03.

\subsubsection{Die Semantik des Konzepts Variation}

Das Konzept VARIATION spielt sowohl bei GP 01 als auch bei GP 02 eine Rolle. GP 01 unterscheidet in zwei verschiedene Arten der Variation: einerseits eine regionale Form (,viele dialekte“) ${ }^{40}$ und andererseits eine internationale Form (wird in verschiedenen ländern gesprochen“, vgl. Abb. 2). Für die regionale Variation werden NORDFRIESISCH und PlatTDEUTSCH als Beispiele genannt. PlatTDEUTSCH wird anschließend positiv bewertet, was die GP auf eine durch die Großmutter bedingte persönliche Beziehung zurückführt. Ihm wird darüber hinaus eine kulturstiftende Funktion zugeschrieben. Im Gegensatz dazu wird das SÄCHSISCHE

40 Die Äußerungen der GP wurden orthographisch in Kleinbuchstaben transkribiert. Dieser Standard wird auch in diesem Beitrag beibehalten. 
negativ bewertet (,wahrscheinlich auch weil es ungewohnt ist“). Das Plattdeutsche weise, so GP 01, keine Form der Standardisierung auf („da gibt es kein richtig kein falsch keine zeichensetzung“, vgl. Abschnitt 4.3.3). Die internationale Variation verbindet GP 01 mit einer Form der Plurizentrizität des Deutschen (,wird in verschiedenen ländern gesprochen also auch schweiz wobei das schweizerisch dann ist aber österreich“, vgl. Kellermeier-Rehbein 2014: 28-33; Schmidlin 2011). Auch in angrenzenden EU-Staaten wie Dänemark, Schweden, den Niederlanden und Polen werde Deutsch gesprochen, in Frankreich hingegen nicht. Außerdem gebe es einen durch Immigration bedingten Sprachkontakt mit dem Russischen und dem Polnischen („da kommen ja auch viele als herkunftsland her polen memel da oben die ecke russland so ein bisschen und von daher die haben ja eben auch die sprache mitgebracht“). Die internationale Dimension der Variation spielt bei GP 02 keine Rolle, er beschränkt sich auf die national-regionale Variation. Er unterscheidet einerseits zwischen regionaler Variation („dialekte“) und andererseits zwischen „eigenständigen Sprachen im deutschen Sprachraum“. Als Beispiel wird das FRIESISCHE genannt, das früher eine höhere Relevanz besessen habe als heute. Die Herausbildung sei historisch bedingt, heute sei es allerdings keine Amtssprache mehr, wodurch die gesunkene Relevanz zu begründen sei. Das DIALEKT-Konzept von GP 02 ist recht ausgeprägt und weist insbesondere für das Niederdeutsche dezidierte Konzeptualisierungen auf (vgl. Abb. 3). Dialekt wird gleich auf zwei Arten von der GP definiert. Einerseits handele es sich um „eine besondere prägung abänderung des hochdeutschen“ und andererseits handelt es sich dabei um „regionen in denen die deutsche sprache anders gesprochen wird als das hochdeutsche die hochdeutsche normsprache“. Diese Definitionen offenbaren eine Ex-negativoKonstruktion von Dialekt als von der Norm bzw. dem Standard abweichend..$^{41}$ Dialekt wird in Hinblick auf die Systemebenen Phonologie (,besondere Aussprache“) und Lexik („,besonderer Wörter und Begriffe“) beschrieben. Darüber hinaus wird die Intonation (,besonderer Tonfall“) im Dialekt hervorgehoben und als anders als im HochDEUTSCHEN markiert. Durch die Herkunft der GP bedingt ist es nicht weiter überraschend, dass das Konzept für das Niederdeutsche am ausgeprägtesten ist (vgl. Abb. 3). Dem Niederdeutschen wird einerseits eine identitätsstiftende Funktion zugeschrieben, andererseits wird auch hier eine abnehmende Relevanz attestiert, was über eine sich verändernde Sozialstruktur erklärt wird, da Niederdeutsch ursprünglich die „sprache der einfachen leute“

41 Die Ex-negativo-Konstruktion wird auch dadurch deutlich, dass die GP das HochDEUTSCHE als durch Dialekt „ein bisschen verhunzt“ beschreibt. Es scheint zusätzlich also Dialektinterferenzen des HOCHDEUTSCHEN zu geben. 
gewesen sei, in der Schule aber nicht gesprochen werden durfte. Dies habe auf lange Sicht zu dem angesprochenen Relevanzverlust geführt. Bemerkenswert ist, dass bei GP 02 ein Bewusstsein über die Diskussion besteht, ob das Niederdeutsche als Dialekt des Deutschen oder als eigenständige Sprache gelten könne. Dies ist möglicherweise als Reliktwissensbestand aus der universitären Ausbildung der GP zu begründen, die ursprünglich als Deutsch-Lehrer ausgebildet wurde, nach dem Studium diesen Weg aber nicht weiterverfolgte. GP 02 verknüpft, trotz diesem Wissen, das Niederdeutsche aber zunächst als Dialekt. Weitere Beispiele der GP sind das SÄCHSISCHE, das BAYERISCHE („,buab und madl“) sowie das HESSISCHE (,,aschebecher; messerstescher; über den blauen block; äbblwoi; babbeln“), bei denen aber neben den wenigen lexikalischen Beispielen keine nähere Charakterisierung erfolgt. Dies zeigt die problematische Einordnung von GP 02 in das traditionelle Verständnis von Experten und Laien (vgl. Hoffmeister 2019), da insbesondere das diachrone Wissen deutlich über prototypische Laienwissensbestände hinausgeht. Allerdings unterscheidet sich GP 02 doch von Experten dahingehend, dass das umfassende Wissen nicht vorhanden ist und die Person nicht mehr in ihrem professionellen Umfeld arbeitet. Insofern liegt es nahe, der traditionellen Laien-Experten-Dichotomie einerseits eine graduelle Kategorienzugehörigkeit mit unscharfen Kategoriengrenzen zu ergänzen, andererseits eine diachrone Komponente miteinzubeziehen, die die Möglichkeit beschreibt, dass ein Expertenstatus durch dynamische Prozesse verlorengeht (oder natürlich erworben wird).

\subsubsection{NORM- und StANDARDISIERUngssemantik}

Die NoRM- und STANDARDISIERUNGssemantik wurde schon in Abschnitt 4.3.1 angedeutet. Sie ist für die Konzeptualisierung von GP 03 grundlegend (vgl. Abb. 4) und auch im Epistemikon von GP 02 relevant (vgl. Abb. 3), da es als Maß für eine dialektale Abweichung genutzt wird („hochdeutsche normsprache“). Sie zeichne sich darüber hinaus durch eine gewisse Merkmalslosigkeit aus (vgl. Beuge 2019: 151-161). Die NORMSPRACHE sei darüber hinaus Ergebnis eines kodifizierten Standards, da sie aus „begriffen und wörtern die in wörterbüchern oder im duden festgelegt sind“ aufgebaut sei (vgl. Beuge 2019: 171-172). Funktional wird eine genormte Standardsprache insbesondere dem Literaturbetrieb sowie dem Bereich Medien und Presse zugeschrieben.

GP 03 konzeptualisiert NoRM auf eine andere Art. Dort geht es weniger um eine Dichotomie NoRM-VARIATION, sondern vielmehr um ein Normideal, an dem sich der Sprachgebrauch orientieren muss, um den Ansprüchen der „richtigen“ 
Sprache zu genügen (,sehr wichtig dass alle richtig deutsch sprechen und schreiben lernen“). Dabei wird immer wieder auf die Kategorie des VERSTEHENS referiert, das als Ankerpunkt für die Legitimation von unterschiedlichen Sprechweisen dient. Es wird die heutige Sprachverwendung von Jugendlichen und Menschen mit Migrationshintergrund besonders kritisiert (vgl. Kilian, Niehr \& Schiewe 2016: 71, sowie 104-111). Von GP 03 werden prinzipiell gewisse Wortpaare kritisiert (z. B. ein Unterschied von ,mir' und ,mich' sowie von ,dasselbe' und ,das gleiche') sowie der Sprachgebrauch im Ganzen („,dieses kauderwelsch"). Diese Verstehensbarriere führt zu einem Normbedürfnis und der widerholten Betonung der Normrelevanz. Besondere Betonung liegt auf der Rechtschreibreform, bei der viele Punkte als „vollkommen unsinnig“ beschrieben werden. Diese Einstellung wird insbesondere am Beispiel der Schreibung von ,mithilfe' beschrieben. Neben der Rechtschreibreform wird die Leichte Sprache als VEREINFACHTE SPRACHE als „,so blödsinnig dass kein mensch begreift was sie meinen" beschrieben (vgl. auch Bock \& Antos 2019: 74-75). Die Aussage „da wird nur wieder alles neu gemacht“ deckt sich mit der Charakteristik laienlinguistischer Sprachkritik als Sprachkonservatismus (vgl. Kilian, Niehr \& Schiewe 2016: 108-110).

Insgesamt unterscheiden sich die beiden Konzeptualisierungen der GP von NORM und STANDARD also, da einerseits eine NORMSPRACHE im Unterschied zu Dialekt konzeptualisiert wird (GP 02) und andererseits ein Normbedürfnis formuliert wird (GP 03). Die Ansätze der jeweils anderen GP spielen in den Konzeptualisierungen keine Rolle.

\subsubsection{Die Semantik des Konzepts Tradition}

Dass der deutschen Sprache eine TRADITION zugeschrieben wird, geschieht in beiden Fällen (GP 02 \& GP 03) über die Beschreibung der Relevanz von Literatur. Bei GP 03 wird die Bedeutung von Literatur einhergehend mit einem Relevanzverlust konzeptualisiert. Heute, so die GP, sei man nicht mehr so interessiert an Gedichten (d.h. insbesondere Goethe und Schiller). Früher habe das eine andere Bedeutung gehabt, da man einerseits interessiert gewesen sei und andererseits Freude empfunden habe, sie zu lernen. Der in Abschnitt 4.3.2 angesprochene Konservatismus kommt hier erneut zum Tragen. Goethe und Schiller sind neben Thomas Mann und Günter Grass auch für die Konzeptualisierung von GP 02 relevant. Ihnen wird als Vertreter des Schriftstellertums Weltruhm und ein verändernder Einfluss auf die Sprache zugeschrieben, da sie deutsche Sprache be- und verarbeiteten. Allerdings erhält das Konzept TRADITION bei GP 
02 eine zweite Komponente und zwar eine diachrone Dimension, da das Deutsche, das sich aus dem Mittelhochdeutschen entwickelt habe (möglicher- weise Reliktwissen, vgl. Abschnitt 4.3.1) über die verschiedenen Sprachstufen bis heute eine lange Tradition nachweisen könne. Diese Tradition wird zwar auch für andere Sprachen vermutet, aber nicht näher expliziert.

\subsection{Interpretative Ansätze}

Im Folgenden sollen die Ergebnisse auf die in Abschnitt 4.1 entwickelten Hypothesen angewendet werden. $\mathrm{Zu}$ bemerken ist, dass die Zuverlässigkeit ob der eingeschränkten Datenmenge nicht vollumfänglich gegeben ist, sodass die Ergebnisse Tendenzen darstellen. Die Anwendung auf die Hypothesen sind demnach ebenfalls als ein erster Aufschlag zu verstehen.

\subsubsection{Die Standardisierungshypothese}

Die Standardisierungshypothese lässt sich in Ansätzen bestätigen, da die drei Konzeptbereiche in Abschnitt 4.3 immer von zwei GP geteilt werden. Insofern sind gewisse Überschneidungen in den Konzeptualisierungen durchaus erkennbar. Um allerdings eine zuverlässige Aussage zu treffen, bedarf es einer größeren Stichprobe, die für Hoffmeister (2020c) vorliegt, für den vorliegenden Artikel allerdings noch nicht ausgewertet werden konnten. Die in Abschnitt 4.1 angesprochene Filler-Varianz zeigt sich insbesondere in Bezug auf die NoRMund STANDARDISIERUNGssemantik.

\subsubsection{Die Komplexitätshypothese}

Die Komplexitätshypothese kann durch die Auswertung der Daten bestätigt werden. Das zeigt sich nicht nur in der Länge der Interviews, sondern insbesondere durch die einzelnen Epistemika (vgl. Abb. 2, 3 und 4). Dort wird nicht nur die divergierende Anzahl an Konzepten deutlich, sondern die unterschiedliche Komplexität zeigt sich vor allem in der Varianz der Verbindungen zwischen den Konzepten. 


\subsubsection{Die Repräsentationshypothese}

Die Repräsentationshypothese lässt sich an dem untersuchten Datenmaterial nicht bestätigen. Es wurden nicht bloß nominale Konzepte repräsentiert, sondern eine diverse Menge an Sprachdaten (i. d. R. in Satzform). Das hat zur Folge, dass die Auswertung und Interpretation der Daten schwieriger und unzuverlässiger wird, da die Interpretationsleistung des Forschers einen größeren Stellenwert einnimmt. Abgesichert und plausibilisiert wird die Interpretation schließlich durch den zweiten Interviewteil, indem die einzelnen reproduzierten Items abgefragt werden und um Erläuterung gebeten wird (vgl. Abschnitt 4.2).

\section{Zusammenfassende Abschlussbemerkungen, Ausblick und weitere Desiderata}

Der vorliegende Beitrag konnte erste Tendenzen in der Konzeptualisierung linguistischer Laien zur deutschen Sprache aufzeigen. Drei Konzepte konnten als dominierend herausgearbeitet werden: VARIATION, NORM bzw. STANDARD und TRADITION. Dabei hat sich gezeigt, dass die GP jeweils eine unterschiedliche Vorstellung von den einzelnen Konzepten haben bzw. neue Aspekte perspektivieren. Während GP 01 in Bezug auf die Variation zwischen einer regionalen und einer internationalen (plurizentrischen) Variation unterschieden hat, spielte dies bei GP 02 keine Rolle. Dort wurde Dialekt als vom Standard abweichend konzeptualisiert. Diese Konzeptualisierung bildet dann auch die Grundlage für Abschnitt 4.3.2, in dem der Standard von GP 02 als merkmalsfrei und in Wörterbüchern kodifiziert abgebildet wird. Für GP 03 existiert ein großes Normbedürfnis, das sich implizit auch im Konzept TRADITION widerspiegelt, da die GP im Rahmen ihres Sprachkonservatismus Bedauern äußert, dass die Literatur, die mit Goethe und Schiller verbunden wird, im Vergleich zu ,früher` an Bedeutung verloren habe. GP 02 schreibt den Schriftstellern einen sprachverändernden Einfluss zu.

Die Ergebnisse können zeigen, dass die in Abschnitt 3.3 dargestellte Theorie des Epistemikons (nebst weiteren Explikationen) vielversprechend und zielführend ist. Die kontinuierliche Reproduktion der Wissensbestände weist darauf hin, dass der Stimulus ,Deutsche Sprache“ für eine primäre Aktivierung von Wissen ausreichend ist. Anschließend erfolgt eine Aktivierungskette anderer Wissensbestände. Dass einiges Wissen zunächst inaktiv ist, zeigt sich, da es im 
zweiten Interviewteil durch die Abfrage der einzelnen Konzepte aktiviert werden konnte. Um Aussagen zu den Kategorisierungslinks machen zu können, muss ein adäquates Untersuchungssetting gewählt werden, dies ist nicht das Ziel der vorliegenden Untersuchung.

Für eine umfassende Theoriebildung bedarf es zukünftig einer intensiven Auseinandersetzung mit framesemantischen Ansätzen, um so zu einer konsistenten, den Ansprüchen der kognitiven Semantik Rechnung tragenden Theorie kommen zu können. Des Weiteren wäre es für das Modell essentiell, etablierte wahrnehmungspsychologische Aspekte, insbesondere der Salienz und Pertinenz (vgl. Purschke 2011), zu integrieren.

Auf empirischer Ebene müssen die Konzepte durch eine umfassendere Datengrundlage weiter ausgebaut und plausibilisiert werden. Für Anschlussstudien wäre zudem eine Untersuchung (und damit Aktivierung) einzelner Sprachkonzepte lohnend. So könnte der Ansatz des Epistemikons genutzt werden, um im Rahmen wahrnehmungsdialektologischer Studien einen ergänzenden Weg einzuschlagen und dem Postulat von Dialekt als kognitives Phänomen (vgl. Sauer \& Hoffmeister i. Vorb.) Rechnung zu tragen.

Als Ergebnis des Beitrages lässt sich also festhalten, was bereits in dem Beitrag vorangestellten Zitat deutlich wurde: „Die Sprache stellt semantische Felder oder Sinnzonen her, die wiederum durch Sprache abgegrenzt werden. Vokabular, Grammatik und Syntax sorgen für Gliederung und Ordnung im Zuständigkeitsbereich der Semantik“ (Berger \& Luckmann 2013: 42). Diese semantischen Felder ${ }^{42}$, die aus einzelnen semantischen Einheiten bestehen, müssen im Rahmen laienlinguistischer Studien weiter untersucht werden, um möglichst ein umfassendes Bild laienlinguistischer Konzeptualisierungen von Sprache zu erhalten.

42 Im Rahmen weiterführender kognitionslinguistischer Studien wäre es zudem lohnend, zu untersuchen, auf welche Konstruktionen und (FrameNet-)Frames von den GP bei der Wiedergabe der Konzepte zugegriffen wird (vgl. exemplarisch Imo \& Ziegler 2019). Im Anschluss könnten diese Einheiten dann mit dem Epistemikon in Verbindung gebracht werden. Für diesen Hinweis danke ich Ann-Katrin Nöhren (Düsseldorf). 


\section{Literaturverzeichnis}

Anders, Christina Ada (2010): Wahrnehmungsdialektologie. Der deutsche Sprachraum aus der Sicht linguistischer Laien. Berlin, New York: De Gruyter.

Ankenbrand, Katrin (2013): Höflichkeit im Wandel. Entwicklungen und Tendenzen in der Höflichkeitspraxis und dem laienlinguistischen Höflichkeitsverständnis der bundesdeutschen Sprachgemeinschaft innerhalb der letzten fünfzig Jahre. Dissertation, Ruprecht-KarlsUniversität Heidelberg.

Antos, Gerd (1996): Laien-Linguistik. Studien zu Sprach- und Kommunikationsproblemen im Alltag. Am Beispiel von Sprachratgebern und Kommunikationstrainings. Tübingen: Niemeyer.

Antos, Gerd, Thomas Niehr \& Jürgen Spitzmüller (Hrsg.) (2019): Handbuch Sprache im Urteil der Öffentlichkeit. Berlin, Boston: De Gruyter.

Becker, Lidia, Sandra Herling \& Holger Wochele (Hrsg.) (i. E.): Manuel de linguistique populaire. Berlin, Boston: De Gruyter.

Bengtsson, Jan (2013): Embodied Experience in Educational Practice and Research. Studies in Philosophy and Education 32 (1), 39-53.

Berger, Peter L. \& Thomas Luckmann (2013): Die gesellschaftliche Konstruktion der Wirklichkeit. 25. Auflage. Frankfurt am Main: Fischer.

Berthele, Raphael (2002): Attitudes and Mental Model of Language: On the Cognitive Foundation of Sociolinguistic Practice. In Gunnstein Akselberg (Hrsg.), Målbryting. Skrifter frå prosleket Talemålsendring i Noreg. Nr. 6: Språkleg identitet og haldning, 25-66. Bergen: Nordisk institutt, Universitetet i Bergen.

Beuge, Patrick (2019): Was ist gutes Deutsch? Eine qualitative Analyse laienlinguistischen Sprachnormwissens. Berlin, Boston: De Gruyter.

Bock, Bettina M. \& Gerd Antos (2019): ,Öffentlichkeit‘ - ,Laien` - ,Experten`: Strukturwandel von ,Laien ' und ,Experten“ in Diskursen über ,Sprache‘. In Gerd Antos, Thomas Niehr \& Jürgen Spitzmüller (Hrsg.), Handbuch Sprache im Urteil der Öffentlichkeit, 54-79. Berlin, Boston: De Gruyter.

Bucholtz, Mary \& Kira Hall (2016): Embodied sociolinguistics. In Nikolas Coupland (Hrsg.), Sociolinguistics. Theoretical Debates, 173-197. Cambridge: Cambridge University Press.

Busse, Dietrich (1987): Historische Semantik. Analyse eines Programms. Stuttgart: Klett Cotta. Busse, Dietrich (2012): Frame-Semantik. Ein Kompendium. Berlin, Boston: De Gruyter.

Busse, Dietrich (2018): Diskurs und Wissensrahmen. In Ingo H. Warnke (Hrsg.), Handbuch Diskurs, 3-29. Berlin, Boston: De Gruyter.

Chomsky, Noam (1957): Syntactic Structures. Den Haag: Mouton.

Chomsky, Noam (1959): Review of Skinners “Verbal Behavior”. Language 35 (1), 26-58.

Coupland, Nikolas \& Adam Jaworski (2004): Sociolinguistic perspectives on metalanguage. Reflexivity, evaluation and ideology. In Adam Jaworski, Nikolas Coupland \& Dariusz Galasiński (Hrsg.), Metalanguage. Social and Ideological Perspectives, 15-51. Berlin, New York: De Gruyter.

Cuonz, Christina (2014): Sprachliche Werturteile von Laien. Eine sozio-kognitive Analyse. Tübingen: Francke.

Deppermann, Arnulf (2015): Pragmatik revisited. In Ludwig M. Eichinger (Hrsg.), Sprachwissenschaft im Fokus. Positionsbestimmungen und Perspektiven, 323-352. Berlin u. a.: De Gruyter. 
Diaz-Bone, Rainer (2013): Sozio-Episteme und Sozio-Kognition. Epistemologische Zugänge zum Verhältnis von Diskurs und Wissen. In Willy Viehöver, Reiner Keller \& Werner Schneider (Hrsg.), Diskurs - Sprache - Wissen. Interdisziplinäre Beiträge zum Verhältnis von Sprache und Wissen in der Diskursforschung, 78-96. Wiesbaden: Springer.

Evans, Vyvyan (2014): The Language Myth. Why Language is not an Instinct. Cambridge: Cambridge University Press.

Faulstich, Katja (2008): Konzepte des Hochdeutschen. Der Sprachnormierungsdiskurs im 18. Jahrhundert. Berlin, New York: De Gruyter.

Felder, Ekkehard (2013): Faktizitätsherstellung mittels handlungsleitender Konzepte und agonaler Zentren. Der diskursive Wettkampf um Geltungsansprüche. In Ekkehard Felder (Hrsg.), Faktizitätsherstellung in Diskursen. Die Macht des Deklarativen, 13-28. Berlin, Boston: De Gruyter.

Felder, Ekkehard (2016): Einführung in die Varietätenlinguistik. Darmstadt: WBG.

Felder, Ekkehard (2018): Wahrheit und Wissen zwischen Wirklichkeit und Konstruktion. Freiheiten und Zwänge beim sprachlichen Handeln. In Ekkehard Felder \& Andreas Gardt (Hrsg.), Wirklichkeit oder Konstruktion? Sprachtheoretische und interdisziplinäre Aspekte einer brisanten Alternative, 371-398. Berlin, Boston: De Gruyter.

Felder, Ekkehard \& Andreas Gardt (Hrsg.) (2018): Wirklichkeit oder Konstruktion? Sprachtheoretische und interdisziplinäre Aspekte einer brisanten Alternative. Berlin, Boston: De Gruyter.

Fillmore, Charles J. (1982): Frame-Semantics. In The Linguistic Society of Korea (Hrsg.), Linguistic in the Morning Calm. Selected Papers from SICOL-1981, 111-137. Seoul: Hanshin.

Fillmore, Charles J. (1985): Frames and the semantics of understanding. Quaderni di Semantica 6 (2), 222-254.

Fingerhuth, Matthias \& Hans C. Boas (2018): Anglizismen zwischen Linguistik und LaienLinguistik: Zum Fremdwortpurismus des Vereins Deutsche Sprache im Anglizismen Index. Eine frame-semantische Analyse seiner Metatexte. In Csaba Földes (Hrsg.), Sprach- und Textkulturen - interkulturelle und vergleichende Konzepte, 19-41. Tübingen: Narr Francke Attempto.

Furnham, Adrian F. (1988): Lay Theories. Everyday understanding of problems in the social sciences. Oxford: Pergamon Press.

Gardt, Andreas (2001): Beeinflusst Sprache unser Denken? Ein Überblick über Positionen der Sprachtheorie. In Andrea Lehr et al. (Hrsg.), Sprache im Alltag. Beiträge zu neuen Perspektiven in der Linguistik. Herbert Ernst Wiegand zum 65. Geburtstag gewidmet, 19-39. Berlin, New York: De Gruyter.

Gautier, Laurent \& Simon Varga (Hrsg.) (i. Vorb.): Frames und Fachwissen.

Gipper, Helmut (1987): Das Sprachapriori. Sprache als Voraussetzung menschlichen Denkens und Erkennens. Stuttgart, Bad Cannstadt: Frommann-Holzboog.

Goldberg, Adele A. (1995): Constructions. A Construction Grammar Approach to Argument Structure. Chicago: University of Chicago Press.

Groeben, Norbert \& Brigitte Scheele (1982): Einige Sprachregelungsvorschläge für die Erforschung Subjektiver Theorien. In Hanns-Dietrich Dann et al. (Hrsg.), Analyse und Modifikation Subjektiver Theorien von Lehrern. Ergebnisse und Analysen eines Kolloquiums, 13-39. Konstanz: Universitätsverlag.

Groeben, Norbert et al. (Hrsg.) (1988): Das Forschungsprogramm Subjektive Theorien. Eine Einführung in die Psychologie des reflexiven Subjekts. Tübingen: Francke Verlag. 
Habermas, Jürgen (1973): Wahrheitstheorien. In Helmut Fahrenbach (Hrsg.), Wirklichkeit und Reflexion. Walter Schulz zum 60. Geburtstag, 211-266. Pfullingen: Neske.

Habermas, Jürgen (1995): Theorie des kommunikativen Handelns. 2 Bde. Frankfurt am Main: Suhrkamp.

Haddock, Geoffrey \& Gregory R. Maio (2014): Einstellungen. In Klaus Jonas, Wolfgang Stroebe \& Miles Hewstone (Hrsg.), Sozialpsychologie. 6. vollst. überarb. Auflage, 197-229. Berlin, Heidelberg: Springer.

Hoenigswald, Henry (1966): A proposal for the study of folk-linguistics. In William Bright (Hrsg.), Sociolinguistics. Proceedings of the UCLA Sociolinguistics Conference, 1964, 1626. The Hague, Paris: De Gruyter Mouton.

Hoffmeister, Toke (2017): Der Einfluss der regionalen Herkunft auf das Dialektwissen linguistischer Laien. In Markus Hundt, Nicole Palliwoda \& Saskia Schröder (Hrsg.), Der deutsche Sprachraum aus der Sicht linguistischer Laien. Ergebnisse des Kieler DFG-Projektes, 213261. Berlin, Boston: De Gruyter.

Hoffmeister, Toke (2019): Laien als Experten und Experten als Laien. Zur Problematik eines etablierten Begriffspaares. Linguistik Online 99 (6), 151-174. http://dx.doi.org/10.13092/lo.99.5969 (letzter Zugriff 10.02.2020).

Hoffmeister, Toke (2020a): Die Aktivierung inaktiver Wissensbestände. Zur Repräsentation dialektologischen Wissens. In Markus Hundt, Andrea Kleene, Albrecht Plewnia \& Verena Sauer (Hrsg.), Regiolekte - objektive Sprachdaten und subjektive Wahrnehmung, 157184. Tübingen: Narr Francke Attempto.

Hoffmeister, Toke (2020b): Subjektive Grammatikalitätstheorien. Entstehung, Verbreitung und forschungspraktische Konsequenzen. Deutsche Sprache 3/2020, 233-248.

Hoffmeister, Toke (2020c): Sprachwelten und Sprachwissen. Theorie und Praxis einer kognitiven Laienlinguistik. Dissertation. Philosophische Fakultät der Christian-AlbrechtsUniversität zu Kiel.

Hörmann, Hans (1978): Meinen und Verstehen. Grundzüge einer psychologischen Semantik. Frankfurt am Main: Suhrkamp.

Hundt, Markus (2017): Struktur und Komplexität des linguistischen Laienwissens. In Markus Hundt, Saskia Schröder \& Nicole Palliwoda (Hrsg.), Der deutsche Sprachraum aus der Sicht linguistischer Laien. Ergebnisse des Kieler DFG-Projektes, 121-159. Berlin, Boston: De Gruyter.

Hundt, Markus (2018): Wahrnehmungsdialektologie - quo vadis? In Alexandra Lenz \& Albrecht Plewnia (Hrsg.), Variation - Norm(en) - Identitä(en), 99-126. Berlin, Boston: De Gruyter. Hundt, Markus, Nicole Palliwoda \& Saskia Schröder (Hrsg.) (2017): Der deutsche Sprachraum aus der Sicht linguistische Laien. Ergebnisse des Kieler DFG-Projektes. Berlin, Boston: De Gruyter.

Husserl, Edmund (1976): Ideen zu einer reinen Phänomenologie und phänomenologischen Philosophie. Buch 1. Allgemeine Einführung in die reine Phänomenologie. Halbband 1. Hg. v. Karl Schumann. Den Haag: Martinus Nijhoff.

Imo, Wolfgang \& Evelyn Ziegler (2019): Situierte Konstruktionen: das Indefinitpronomen man im Kontext der Aushandlung von Einstellungen zu migrationsbedingter Mehrsprachigkeit. In Sabine de Knop \& Jürgen Erfurt (Hrsg.), Konstruktionsgrammatik und Mehrsprachigkeit, 75-104. Duisburg: Universitätsverlag Rhein-Ruhr.

Jung, Carl Gustav (1979): Experimentelle Untersuchungen. Olten: Walter. 
Kalverkämper, Hartwig (1990): Gemeinsprache und Fachsprachen: Plädoyer für eine integrierende Sichtweise. In Gerhard Stickel (Hrsg.), Deutsche Gegenwartssprache: Tendenzen und Perspektiven, 88-133. Berlin, New York: De Gruyter.

Karliczek, Anja (2019): Wir wollen Bürgerwissenschaften als selbstverständliche Forschungsmethode etablieren. Pressemitteilung 122/2019. Bundesministerium für Bildung und Forschung. https://www.bmbf.de/de/karliczek-wir-wollen-buergerwissenschaften-alsselbstverstaendliche-forschungsmethode-9930.html (letzter Zugriff 10.02.2020).

Kellermeier-Rehbein, Birte (2014): Plurizentrik. Einführung in die nationalen Varietäten des Deutschen. Berlin: Erich Schmidt.

Kienpointner, Manfred (1992): Alltagslogik. Struktur und Funktion von Argumentationsmustern. Stuttgart-Bad Cannstatt: Fromann-Holzboog.

Kilian, Jörg, Thomas Niehr \& Jürgen Schiewe (2016): Sprachkritik. Ansätze und Methoden der kritischen Sprachbetrachtung. 2. Auflage. Berlin, Boston: De Gruyter.

Klann-Delius, Gisela (2016): Spracherwerb. Eine Einführung. 3. aktual. und erw. Auflage. Stuttgart: Metzler.

Konerding, Klaus-Peter (2001): Sprache im Alltag und kognitive Linguistik. Stereotype und schematisiertes Wissen. In Andrea Lehr et al. (Hrsg.), Sprache im Alltag. Beiträge zu neuen Perspektiven in der Linguistik. Herbert Ernst Wiegand zum 65. Geburtstag gewidmet, 151-172. Berlin, New York: De Gruyter.

Konerding, Klaus-Peter (2015): Sprache und Wissen. In Ekkehard Felder \& Andreas Gardt (Hrsg.), Handbuch Sprache und Wissen, 57-80. Berlin, Boston: De Gruyter.

Kotthoff, Helga \& Damaris Nübling (2018): Genderlinguistik. Eine Einführung in Sprache, Gespräch und Geschlecht. Unter Mitarbeit von Claudia Schmidt. Tübingen: Narr Francke Attempto.

Köller, Wilhelm (2004): Perspektivität und Sprache. Zur Struktur von Objektivierungsformen in Bildern, im Denken und in der Sprache. Berlin, New York: De Gruyter.

König, Katharina (2014): Spracheinstellungen und Identitätskonstruktion. Eine gesprächsanalytische Untersuchung sprachbiographischer Interviews mit Deutsch-Vietnamesen. Berlin: Akademie.

Kremer, Ludger (1984): Die niederländisch-deutsche Staatsgrenze als subjektive Dialektgrenze. In Nedersaksisch Instituut (Hrsg.), Grenzen en Grensproblemen. Een bundel studies uitgegeven door het Nedersaksisch Instituut van de R.U. Groningen ter gelegenheid van zijn 30-jarig bestaan, 76-83. Groningen: Sasland.

Langacker, Ronald W. (1987): Foundations of Cognitive Grammar. Volume I: Theoretical Prerequisites. Stanford: Stanford University Press.

Lasch, Alexander (2016): Nonagentive Konstruktionen des Deutschen. Berlin, Boston: De Gruyter.

Lehr, Andrea (2002): Sprachbezogenes Wissen in der Lebenswelt des Alltags. Tübingen: Niemeyer.

Link, Jürgen (2005): Warum Diskurse nicht von personalen Subjekten ,ausgehandelt‘ werden. Von der Diskurs- zur Interdiskurstheorie. In Reiner Keller, Andreas Hirseland, Werner Schneider \& Willy Viehöver (Hrsg.), Die diskursive Konstruktion von Wirklichkeit. Zum Verhältnis von Wissenssoziologie und Diskursforschung, 77-99. Konstanz: UVK.

Mattheier, Klaus (1985): Dialektologie der Dialektsprecher. Überlegungen zu einem interpretativen Ansatz in der Dialektologie. Germanistische Mitteilungen 21, 47-67. 
Möller, Robert \& Stephan Elspaß (2015): Atlas zur deutschen Alltagssprache (AdA). In Roland Kehrein, Alfred Lameli \& Stefan Rabanus (Hrsg.), Regionale Variation des Deutschen. Projekte und Perspektiven, 519-539. Berlin, Boston: De Gruyter.

Palliwoda, Nicole (2019): Das Konzept Mauer in den Köpfen. Der Einfluss der Priming-Methode auf die Sprechprobenverortung und -bewertung. Stuttgart: Steiner.

Paul, Ingwer (1999): Praktische Sprachreflexion. Tübingen: Niemeyer.

Paul, Ingwer (2003): Subjektive Sprachtheorien von Lehrenden und Lernenden. In Ursula Bredel et al. (Hrsg.), Didaktik der deutschen Sprache. Ein Handbuch. 2. Teilband, 650657. Paderborn u. a.: Schöningh.

Peters, Bernhard (2007): Der Sinn von Öffentlichkeit. Frankfurt am Main: Suhrkamp.

Polanyi, Michael (1985): Implizites Wissen. Frankfurt am Main: Suhrkamp.

Preston, Dennis R. (1982): Perceptual dialectology: Mental maps of United States dialects from a Hawaiian perspective. Working Papers in Linguistics 14 (2), 5-49.

Preston, Dennis R. (2004): Folk Metalanguage. In Adam Jaworski, Nikolas Coupland \& Dariusz Galasiński (Hrsg.), Metalanguage. Social and Ideological Perspectives, 75-101. Berlin, New York: De Gruyter.

Proctor, Robert W. \& Kim-Phuong L. Vu (2006): Stimulus-Response Compatibility Principles. Data, Theory, and Application. Boca Raton, FL: CRC.

Purschke, Christoph (2011): Regionalsprache und Hörerurteil. Grundzüge einer perzeptiven Variationslinguistik. Stuttgart: Steiner.

Purschke, Christoph \& Philipp Stoeckle (2019): Perzeptionslinguistik arealer Sprachvariation im Deutschen. In Joachim Herrgen \& Jürgen Erich Schmidt (Hrsg.), Sprache und Raum Deutsch. Ein internationales Handbuch der Sprachvariation, 844-860. Berlin, Boston: De Gruyter.

Sauer, Verena (2018): Dialektgrenzen - Grenzdialekte. Die Struktur der itzgründischen Dialektlandschaft an der ehemaligen deutsch-deutschen Grenze. Berlin, Boston: De Gruyter.

Sauer, Verena \& Toke Hoffmeister (i. Vorb.): Wahrnehmungsdialektologie. Eine Einführung. Berlin, Boston: De Gruyter.

Schiewe, Jürgen (2004): Öffentlichkeit. Entstehung und Wandel in Deutschland. Paderborn u. a.: Schöningh.

Schmidlin, Regula (2011): Die Vielfalt des Deutschen. Standard und Variation. Gebrauch, Einschätzung und Kodifizierung einer plurizentrischen Sprache. Berlin, Boston: De Gruyter.

Schröder, Saskia (2019): Sprachräumliche Praxis. Sprachraumkartierung in der Wahrnehmungsdialektologie. Frankfurt am Main: Peter Lang.

Schütz, Alfred \& Thomas Luckmann (2003): Strukturen der Lebenswelt. Konstanz: UTB.

Searle, John R. (1997): Die Konstruktion der gesellschaftlichen Wirklichkeit. Zur Ontologie sozialer Tatsachen. Reinbek bei Hamburg: Rowohlt.

Searle, John R. (2012): Wie wir die soziale Welt machen. Die Struktur der menschlichen Zivilisation. Aus dem Amerikanischen von Joachim Schulte. Frankfurt am Main: Suhrkamp.

Skinner, Burrhus F. (1957): Verbal Behavior. New York, NY: Appleton-Century-Crofts.

Spitzmüller, Jürgen (2005): Metasprachdiskurse. Einstellungen zu Anglizismen und ihre wissenschaftliche Rezeption. Berlin, New York: De Gruyter.

Spitzmüller, Jürgen (2009): Metasprachliches Wissen diesseits und jenseits der Linguistik. In Tilo Weber \& Gerd Antos (Hrsg.), Typen von Wissen. Begriffliche Unterscheidung und 
Ausprägungen in der Praxis des Wissenstransfers, 112-126. Frankfurt am Main: Peter Lang.

Spitzmüller, Jürgen (2013): Metapragmatik, Indexikalität, soziale Registrierung. Zur diskursiven Konstruktion sprachideologischer Positionen. Zeitschrift für Diskursforschung 3, 263-287.

Spitzmüller, Jürgen (2019): ,Sprache‘ - ,Metasprache‘ - ,Metapragmatik‘: Sprache und sprachliches Handeln als Gegenstand sozialer Reflexion. In Gerd Antos, Thomas Niehr \& Jürgen Spitzmüller (Hrsg), Handbuch Sprache im Urteil der Öffentlichkeit, 11-30. Berlin, Boston: De Gruyter.

Spitzmüller, Jürgen \& Ingo H. Warnke (2011): Diskurslinguistik. Eine Einführung in Theorien und Methoden der transtextuellen Sprachanalyse. Berlin, Boston: De Gruyter.

Stefanowitsch, Anatol (2011): Konstruktionsgrammatik und Grammatiktheorie. In Alexander Lasch \& Alexander Ziem (Hrsg.), Konstruktionsgrammatik III. Aktuelle Fragen und Lösungsansätze, 11-25. Tübingen: Stauffenburg.

Strauss, Lina (2018): Rhetorikratgeber als Beispiel für die Laienlinguistik. Eine Diskursanalyse. Berlin: Metzler.

Waldenfels, Bernhard (1985): In den Netzen der Lebenswelt. Frankfurt am Main: Suhrkamp. Warnke, Ingo H. (2009): Die sprachliche Konstituierung von geteiltem Wissen in Diskursen. In Ekkehard Felder \& Marcus Müller (Hrsg.), Wissen durch Sprache. Theorie, Praxis und Erkenntnisinteresse des Forschungsnetzwerks „Sprache und Wissen“, 113-140. Berlin, New York: De Gruyter.

Warnke, Ingo H. (Hrsg.) (2018): Handbuch Diskurs. Berlin, Boston: De Gruyter.

Wichter, Sigurd \& Gerd Antos (Hrsg.) (2001): Wissenstransfer zwischen Experten und Laien. Umriss einer Transferwissenschaft. Frankfurt a. M. u. a.: Peter Lang.

Williams, Cara Penry (2020): Folk Linguistics and Social Meaning in Australian English. London, New York: Routledge.

Wilton, Antje \& Martin Stegu (2011): Bringing the ,folk' into applied linguistics. An introduction. In Antje Wilton \& Martin Stegu (Hrsg.), Applied Folk Linguistics. AlLA Review 24, 1-14. Amsterdam: John Benjamin.

Wittgenstein, Ludwig (1921/2016): Tractatus logico-philosophicus. In Ludwig Wittgenstein Werkausgabe Band 1. 22. Auflage, 7-85. Frankfurt am Main: Suhrkamp.

Ziem, Alexander (2008): Frames und sprachliches Wissen. Kognitive Aspekte der semantischen Kompetenz. Berlin, New York: De Gruyter.

Ziem, Alexander (2009): Sprachliche Wissenskonstitution aus Sicht der Kognitiven Grammatik und Konstruktionsgrammatik. In Ekkehard Felder \& Marcus Müller (Hrsg.), Wissen durch Sprache. Theorie, Praxis und Erkenntnisinteresse des Forschungsnetzwerkes „Sprache und Wissen“, 171-204. Berlin, New York: De Gruyter.

Ziem, Alexander (2013): Wozu Kognitive Semantik? In Dietrich Busse \& Wolfgang Teubert (Hrsg.), Linguistische Diskursanalyse. Neue Perspektiven, 217-242. Wiesbaden: Springer.

Ziem, Alexander (2014): Konstruktionsgrammatische Konzepte eines Konstruktikons. In Alexander Lasch \& Alexander Ziem (Hrsg.), Grammatik als Netzwerk von Konstruktionen. Sprachwissen im Fokus der Konstruktionsgrammatik, 15-34. Berlin: De Gruyter.

Ziem, Alexander (2020): Wortbedeutungen als Frames. Ein Rahmenmodell zur Analyse lexikalischer Bedeutungen. In Sven Staffeldt \& Jörg Hagemann (Hrsg.), Semantiktheorien II. Analysen von Wort- und Satzbedeutungen im Vergleich, 27-56. Tübingen: Stauffenburg. 
Ziem, Alexander \& Alexander Lasch (2013): Konstruktionsgrammatik. Konzepte und Grundlagen gebrauchsbasierter Ansätze. Berlin, Boston: De Gruyter. 